\title{
Probing the mass-loss history of AGB and red supergiant stars from $C O$ rotational line profiles
}

\section{Theoretical model - Mass-loss history unravelled in VY CMa}

\author{
L. Decin ${ }^{1,2, \star}$, S. Hony ${ }^{1}$, A. de Koter $^{2}$, K. Justtanont ${ }^{3}$, A. G. G. M. Tielens ${ }^{4,5, \star \star}$, and L. B. F. M. Waters ${ }^{1,2}$ \\ ${ }^{1}$ Department of Physics and Astronomy, Institute for Astronomy, KU Leuven, Celestijnenlaan 200B, 3001 Leuven, Belgium \\ e-mail: Leen. Decin@ster. kuleuven. ac. be \\ 2 Sterrenkundig Instituut Anton Pannekoek, University of Amsterdam, Kruislaan 403, 1098 Amsterdam, The Netherlands \\ 3 Stockholm Observatory, AlbaNova University Center, 10691 Stockholm, Sweden \\ ${ }^{4}$ Kapteyn Astronomical Institute, PO Box 800, 9700 AV Groningen, The Netherlands \\ 5 SRON, PO Box 800, 9700 AV Groningen, The Netherlands
}

Received 17 March 2005 / Accepted 9 June 2006

\section{ABSTRACT}

\begin{abstract}
Context. Mass loss plays a dominant role in the evolution of low mass stars while they are on the Asymptotic Giant Branch (AGB). The gas and dust ejected during this phase are a major source in the mass budget of the interstellar medium. Recent studies have pointed towards the importance of variations in the mass-loss history of such objects.

Aims. By modelling the full line profile of low excitation CO lines emitted in the circumstellar envelope, we can study the mass-loss history of AGB stars.

Methods. We have developed a non-LTE radiative transfer code, which calculates the velocity structure and gas kinetic temperature of the envelope in a self-consistent way. The resulting structure of the envelope provides the input for the molecular line radiative calculations which are evaluated in the comoving frame. The code allows for the implementation of modulations in the mass-loss rate. This code has been benchmarked against other radiative transfer codes and is shown to perform well and efficiently.

Results. We illustrate the effects of varying mass-loss rates in case of a superwind phase. The model is applied to the well-studied case of VY CMa. We show that both the observed integrated line strengths as the spectral structure present in the observed line profiles, unambiguously demonstrate that this source underwent a phase of high mass loss $\left(\sim 3.2 \times 10^{-4} M_{\odot} \mathrm{yr}^{-1}\right)$ some $1000 \mathrm{yr}$ ago. This phase took place for some $100 \mathrm{yr}$, and was preceded by a low mass-loss phase $\left(\sim 1 \times 10^{-6} M_{\odot} \mathrm{yr}^{-1}\right)$ taking some $800 \mathrm{yr}$. The current mass-loss rate is estimated to be in the order of $8 \times 10^{-5} M_{\odot} \mathrm{yr}^{-1}$.

Conclusions. In this paper, we demonstrate that both the relative strength of the CO rotational line profiles and the (non)-occurrence of spectral structure in the profile offer strong diagnostics to pinpoint the mass-loss history.
\end{abstract}

Key words. line: profiles - radiative transfer - stars: AGB and post-AGB - stars: circumstellar matter - stars: mass loss stars: individual: VY CMa

\section{Introduction}

When low and intermediate mass stars approach the end of their lives and ascend the Asymptotic Giant Branch (AGB), mass loss dominates the subsequent evolution ultimately leading to the removal of the entire envelope. In this process, the star develops a dust-driven wind with a velocity of $10-15 \mathrm{~km} \mathrm{~s}^{-1}$ (Habing \& Olofsson 2003, and references therein). AGB stars lose 35-85 per cent of their mass through the stellar wind before ending up as a white dwarf (Marshall et al. 2004). Likewise, more massive stars $\left(M_{\mathrm{ZAMS}} \gtrsim 8 M_{\odot}\right)$ may pass through a red supergiant phase and lose mass in a similar manner.

The mass-loss properties change with time as these stars ascend the AGB and become more luminous. It is often postulated and in rare cases observed (e.g. Justtanont et al. 1996; van Loon et al. 2003; Marshall et al. 2004) that the AGB evolution ends in a very high mass-loss phase, the so-called superwind phase

* Postdoctoral Fellow of the Fund for Scientific Research, Flanders.

$\star \star$ Current address: MS 245-3, NASA Ames Research Center, Moffett Field, CA 94035-1000, USA.
(Iben \& Renzini 1983). The nature and onset of such a superwind are far from understood.

This superwind phase is not the only type of variable mass loss. Recent observations of (post) AGB objects and Planetary Nebulae $(\mathrm{PNe})$ show that winds from late type stars are far from being smooth. The shell structures found around e.g. CRL 2688 (the Egg Nebula, Ney et al. 1975; Sahai et al. 1998), NGC 6543 (the Cat's Eye Nebula, Harrington \& Borkowski 1994) and the AGB star IRC +10 216 (Mauron \& Huggins 1999, 2000; Fong et al. 2003), indicate that the outflow has quasi-periodic oscillations, with density contrasts corresponding to mass-loss variations of up to a factor $\sim 100-1000$ (Schöier et al. 2005). The time scale for these oscillations is typically a few hundred years, i.e. too long to be a result of stellar pulsation, which has a period of a few hundred days, and too short to be due to nuclear thermal pulses, which occur once in ten thousand to hundred thousand years. Presently, the physical mechanism responsible for these variations, their rate of occurrence and their importance in terms of the amount of ejected matter involved are unknown. Possibly they are linked to the hydrodynamical properties of a dust-driven wind. For example, Simis et al. (2001) find 
that a feedback mechanism between the wind-acceleration and dust growth at the base of the wind may cause modulations in the mass-loss rate.

Already in the late seventies, low excitation rotational carbon-monoxide ( $\mathrm{CO}$ ) emission lines emerging from the circumstellar envelopes (CSEs) around AGBs were observed and analysed to estimate the mass-loss rates during the AGB phase (e.g. Zuckermann et al. 1977; Morris 1980; Knapp et al. 1980, 1982; Knapp \& Morris 1985). CO has proven to be instrumental for this since (almost) all the elemental carbon is locked up in $\mathrm{CO}$ throughout most of the envelope. While previous studies have assumed a constant mass-loss rate in the analysis of rotational CO lines, the goal of our study is to exploit the rotational CO line profiles of different excitation levels as a complementary diagnostic to probe variations in mass-loss rate. With the arrival of HIFI and PACS the powerful spectrometers on the Herschel Space Observatory - ESA's far infrared mission to be launched in 2008 - and ALMA - the submillimeter interferometer which is being built by ESO and NRAO in Chile - new high-quality data of $\mathrm{CO}$ emission lines will put new light on this ongoing research of understanding the geometry, physical conditions and mass-loss history of AGB stars.

In this paper, we describe our non-LTE (non-Local Thermo dynamic Equilibrium) radiative transfer code developed to predict the $\mathrm{CO}$ molecular line strengths. In Sect. 2, the equations governing the temperature and velocity structure in the CSE are presented. Since the CO rotational line profiles are very sensitive to the temperature structure in the CSE, the kinetic temperature is calculated self-consistently taking the main heating and cooling processes into account (Sect. 2.2). The code can treat any density profile, allowing us to deduce the amplitude of the massloss variations (Sect. 2.4). The non-LTE radiative transfer code is described in Sect. 3, and the effect of variations in mass loss on the predicted CO profiles is studied. In Sect. 4, we apply our theoretical code to model the observed $\mathrm{CO}$ rotational emission lines in the supergiant VY CMa. We summarise the results in Sect. 5.

\section{Theoretical model for the temperature and velocity structure in the CSE}

A detailed understanding of the wind around red giants requires the solution of the equations of hydrodynamics, specifically in the complex region starting from the stellar surface into the region of dust formation. The equations should include the conservation laws of mass, momentum, and energy, in combination with a chemical model describing the gas phase chemistry in the CSE. In the absence of such a theory, we follow previous work, and focus on those equations describing the velocity and temperature structure in the CSE. The main assumption is a spherically symmetric mass loss. The code builds on the work of Justtanont et al. (1994, hereafter referred to as J94). Main changes are the inclusion of new and improved heating and cooling rates (Sect. 2.2), and the implementation of a variable mass loss (Sect. 2.4). Helium, in addition to atomic and molecular hydrogen, is now also included as collision partner.

\subsection{The gas and dust velocity}

In this study, we will focus on red giants with outflow velocities in excess of $5 \mathrm{~km} \mathrm{~s}^{-1}$, and mass-loss rates larger than $3 \times$ $10^{-7} M_{\odot} \mathrm{yr}^{-1}$. These winds are believed to be driven by radiation pressure on the dust which condenses in the outer parts of the atmosphere (Winters et al. 2003). In this situation, the equations expressing the conservation of mass and momentum are respectively given by (e.g. Goldreich \& Scoville 1976; Tielens 1983, hereafter referred to as GS76 and T83 respectively)

$$
\frac{\mathrm{d} M(r)}{\mathrm{d} t}=\dot{M}(r)=4 \pi r^{2} \rho(r) v(r)
$$

and

$v(r) \frac{\mathrm{d} v(r)}{\mathrm{d} r}=(\Gamma(r)-1) \frac{G M_{\star}}{r^{2}}$,

where $\dot{M}(r)$ refers to the gas mass-loss rate at a radial distance $r$ from the star, $\rho(r)$ is the gas density, $v(r)$ the gas velocity, $M_{\star}$ the stellar mass, and $\Gamma(r)$ the ratio of the radiation pressure force on the dust to the gravitational force.

The total dust mass-loss rate, $\dot{M}_{\mathrm{d}}$, can be written as (J94)

$$
\begin{aligned}
\dot{M}_{\mathrm{d}}(r) & =\int \dot{M}_{\mathrm{d}}(a, r) \mathrm{d} a \\
& =\int A(r) a^{-3.5} n_{\mathrm{H}}(r) \frac{4}{3} \pi a^{3} \rho_{\mathrm{s}} 4 \pi r^{2}\left[v(r)+v_{\mathrm{drift}}(a, r)\right] \mathrm{d} a
\end{aligned}
$$

where $\dot{M}_{\mathrm{d}}(a, r)$ represents the dust mass-loss rate of a grain of size $a, n_{\mathrm{H}}$ the hydrogen number density, $\rho_{\mathrm{s}}$ the specific density of dust and $v_{\text {drift }}(a, r)$ the drift velocity of a grain of size $a$. For the dust composition, we use silicate grains with $\rho_{\mathrm{s}}=3.3 \mathrm{~g} \mathrm{~cm}^{-3}$ (Draine \& Lee 1984). We adopt a grain-size distribution $n_{\mathrm{d}}(a, r) \mathrm{d} a=A(r) a^{-3.5} n_{\mathrm{H}}(r) \mathrm{d} a$, with a minimum grain-size, $a_{\min }$, of $0.005 \mu \mathrm{m}$ and a maximum grain-size, $a_{\max }$, of $0.25 \mu \mathrm{m}$. The slope -3.5 is typical for interstellar dust (Mathis et al. 1977). It is unclear whether this slope is representative for circumstellar dust shells ${ }^{1}$. Studies on dust condensation and growth suggest that the circumstellar dust grain size distribution could be much steeper (Dominik et al. 1989). However, shattering by grain-grain collisions could lead to a much shallower power law (Biermann \& Harwit 1980). The quantity $A(r)$ represents an abundance scale factor giving the number of dust particles in units of particles per $\mathrm{H}$ atom. For the ISM, $A$ is a constant, estimated to be $10^{-25.10} \mathrm{~cm}^{3.5} / \mathrm{H}$ for silicate grains and $10^{-25.13} \mathrm{~cm}^{3.5} / \mathrm{H}$ for carbon grains (Draine \& Lee 1984). In the case of CSEs around giants, the value of $A(r)$ may differ from the ISM value, since it incorporates the (unknown) dust-to-gas mass ratio $(\psi)$ of the CSE. Moreover $A(r)$ varies as a function of the radius $r$ approximately as $v(r) /\left(v(r)+v_{\text {drift }}(r)\right)$. In case of a constant mass-loss rate, $A$ decreases rapidly at the base of the envelope w.r.t. the value when the dust first condenses and the dust velocity is still zero, as the dust is rapidly accelerated by radiation pressure, yielding a lower dust abundance. It then increases until it reaches a constant value when the ratio of $v(r)$ to $\left(v(r)+v_{\text {drift }}(r)\right)$ is constant. $A(r)$ is calculated from the adopted gas mass-loss rate $\dot{M}$ and dust-to-gas mass ratio $\psi$ through Eq. (3).

Considering the motion of grains, and equating the radiation pressure force with the gas drag force, the drift velocity of a grain

\footnotetext{
1 The choice of slope has very little direct influence on the results. The calculated gas-to-dust ratio changes to some extent since the absorption cross-sections are in the Rayleigh regime, and hence scale with the total dust volume. The gas-grain drag on the other hand scales with the geometric cross section. The grain-size distribution also affects the temperature structure through the gas-grain collisional heating. Since smaller grains obtain a smaller drift velocity a steeper grain-size distribution causes less efficient heating. On the other hand, photoelectric yields will be higher for small grains.
} 
of size $a$ can be calculated to be (Kwok 1975; Truong-Bach et al. 1991)

$v_{\text {drift }}(a, r)=v_{K}(a, r)\left[\sqrt{1+x(r)^{2}}-x(r)\right]^{1 / 2}$,

with

$v_{K}(a, r)=\sqrt{\frac{v(r)}{\dot{M}(r) c} \int Q_{\lambda}(a) L_{\lambda} \mathrm{d} \lambda}$

$x(r)=\frac{1}{2}\left[\frac{v_{T}(r)}{v_{K}(r)}\right]^{2}$, and

$v_{T}(r)=\frac{3}{4}\left[\frac{3 k T(r)}{\mu m_{\mathrm{H}}}\right]^{1 / 2}$

with $Q_{\lambda}(a)$ the extinction efficiency as derived by Justtanont \& Tielens (1992) for silicates, $L_{\lambda}$ the monochromatic stellar luminosity at wavelength $\lambda, v_{T}(r)$ the Maxwellian velocity, $T(r)$ the temperature, $k$ the Boltzmann constant, $\mu$ the mean molecular weight of the gas and $m_{\mathrm{H}}$ the mass of the hydrogen atom. We note that the $\mathrm{J} 94$ assumption of $v_{\text {drift }}(a, r)=v_{K}(a, r)$ holds only in the cool outer region where thermal velocities $v_{T}$ are small compared to $v_{\text {drift }}$. The value of $v_{\text {drift }}(a, r)$ should be lower than $\sim 20 \mathrm{~km} \mathrm{~s}^{-1}$, at which sputtering of the dust grains starts to become important (Kwok 1975). When the star is luminous and goes through a phase of low mass loss, this value can be higher than $20 \mathrm{~km} \mathrm{~s}^{-1}$ for a certain grain-size $a$. When this occurs, dust grains of size $a$ are destroyed, and hence the contributions of the dust grain of size $a$ to the gas-grain collisional heating, the photoelectric heating from dust grains and the heat exchange between dust and gas (see Sect. 2.2) is zero.

Using the above-mentioned equations, the ratio of the drag force to the gravitational force $\Gamma(r)$ can be written as (J94)

$$
\Gamma(r)=\frac{3 v(r)}{16 \pi \rho_{\mathrm{s}} c G M_{\star} \dot{M}(r)} \iint \frac{Q_{\lambda}(a) L_{\lambda} \dot{M}_{\mathrm{d}}(a, r)}{a\left[v(r)+v_{\mathrm{drift}}(a, r)\right]} \mathrm{d} \lambda \mathrm{d} a .
$$

\subsection{The thermal balance equation of the gas}

From the first law of thermodynamics expressing the conservation of energy of the gas, the perfect gas law and the equation of mass conservation, the kinetic temperature of the gas in the CSE is governed by the relation

$$
\begin{aligned}
& \frac{1}{T(r)} \frac{\mathrm{d} T(r)}{\mathrm{d} r}=-\frac{4}{3 r}\left(1+\frac{\epsilon(r)}{2}\right) \\
& +\frac{2}{3} \frac{H(r)-C(r)}{k T(r) v(r) n\left(\mathrm{H}_{2}\right)(r)\left[f_{\mathrm{H}}(r)+1+f_{\mathrm{He}}(r)\left(f_{\mathrm{H}}(r)+2\right)\right]}
\end{aligned}
$$

where $\epsilon(r)=\mathrm{d} \ln v(r) / \mathrm{d} \ln r$ is the logarithmic velocity gradient, $H(r)$ the total heating rate per unit volume, $C(r)$ the total cooling rate per unit volume, $f_{\mathrm{H}}(r)$ the number fraction of atomic to molecular hydrogen i.e. $n(\mathrm{H})(r) / n\left(\mathrm{H}_{2}\right)(r)$, and $f_{\mathrm{He}}(r)$ the helium abundance i.e. $n_{\mathrm{He}}(r) / n_{\mathrm{H}}(r)=n(\mathrm{He})(r) /\left(n(\mathrm{H})(r)+2 n\left(\mathrm{H}_{2}\right)(r)\right)$. The first term on the right hand side of Eq. (7) represents the cooling due to adiabatic expansion in case of constant mass loss. The second term represents the balance of the heating and collision-driven radiative cooling processes. The denominator in this expression takes the contribution of the different collision partners $\mathrm{H}, \mathrm{H}_{2}$, and $\mathrm{He}$ into account.

In order to calculate the gas kinetic temperature as a function of radius, all the major heating and cooling sources must be taken into account. The main processes are discussed below. Newly implemented energy sources w.r.t. J94 are photoelectric heating from dust grains, heat exchange between dust and gas, and heating by cosmic rays. All the other heating/cooling terms underwent updates mainly concerning the used inelastic collision rates. As demonstrated by J94, cooling by vibrational excitation of $\mathrm{H}_{2} \mathrm{O}$ molecules is unimportant, since the critical density of $\mathrm{H}_{2} \mathrm{O}$ is too low in the envelope to play a role in the energy balance. We therefore neglect this effect. We note that we have not yet accounted for fine-structure line cooling. This process may be important for supergiants having a strong chromosphere (e.g. $\alpha$ Ori, Rodgers \& Glassgold 1991). A proper calculation of the number densities of the main atoms and molecules requires the implementation of a chemical reaction network programme, which is planned for the future.

\subsubsection{Gas-grain collisional heating}

Both gaseous and dusty material in the CSE are accelerated away from the central star by radiation pressure. This radiation pressure mainly acts on the dust grains, and momentum is transferred to the gas by collisions between gas molecules and dust grains (GS76). These collisions are the main heat source for the gas. The viscous heat input per unit volume can be written as

$H_{\mathrm{gg}}(r)=n_{\mathrm{d}}(a, r) \sigma_{\mathrm{d}}(a) v_{\mathrm{drift}}(a, r) \times \frac{1}{2} \rho(r) v_{\mathrm{drift}}^{2}(a, r)$,

with $\sigma_{\mathrm{d}}(a)$ the geometrical cross section of a dust grain of size $a$, being $\pi a^{2}$. Taking the grain distribution into account, one obtains

$$
\begin{aligned}
H_{\mathrm{gg}}(r)= & \frac{\pi}{2} \times A(r) \times m_{\mathrm{H}} \times n^{2}\left(\mathrm{H}_{2}\right)(r) \times\left(f_{\mathrm{H}}(r)+2\right)^{2} \\
& \times\left(1+4 f_{\mathrm{He}}(r)\right) \times \int a^{-1.5} v_{\mathrm{drift}}^{3}(a, r) \mathrm{d} a,
\end{aligned}
$$

with $m_{\mathrm{H}}$ the mass of the hydrogen atom. Note that viscous heating depends strongly on the value of $v_{\text {drift }}(a, r)$.

\subsubsection{Photoelectric heating from dust grains}

Simple physical arguments demonstrate that the impact of far ultraviolet (UV) radiation (with wavelengths between 912 and $1200 \AA$ ) removes electrons from dust grains in such an efficient way that about $4 \%$ of radiative energy can be converted into kinetic energy, heating the gas (Tielens \& Hollenbach 1985). The calculation of the heating rate of the gas by this process is complicated by the fact that the electron ejection rate and the photoelectron energy depend on the charge of the dust grains, which is a function of temperature $T$ and electron density $n_{\mathrm{e}}$ in the gas. For a single size of grain, this can be incorporated into a semianalytical formalism (de Jong 1977). Dealing with a grain size distribution complicates the computations, since essentially all grains will attain many different ionisation stages under typical conditions. Such single and more highly charged grains all contribute to the photoelectric heating (Bakes \& Tielens 1994).

Computations for the photoelectric ejection of electrons from a dust grain size distribution have only been performed in the carbon-rich case. Bakes \& Tielens (1994) demonstrated that the photoelectric heating rate increases rapidly with decreasing grain size, and "classical" grains $(a \approx 0.1 \mu \mathrm{m})$ contribute negligibly to the total heating rate. Approximately half of the total heating rate is due to species with $a \leq 15 \AA$. The other half is contributed by larger species up to $a \leq 100 \AA$. This decrease in photoelectric efficiency with increasing grain size results partly from the decrease in photoelectric yield, i.e. the photoemission yield of small particles is expected to be significantly larger than 
that of bulk materials, since for bulk materials the photoelectron may lose all its excess energy in collisions with other atoms before it reaches the surface. Furthermore, it is aided by the increase in average grain charge with increasing grain size, which is a direct result of the fact that the UV absorption rate increases with grain volume, while the recombination rate depends only weakly on $a$. Hence, the lower limit of the grain size distribution has a considerable influence on the total photoelectric heating rate, while the upper limit has only little effect.

The physical nature of the circumstellar dust grains around O-rich stars is far less understood and the coupling between the far-UV radiation field and the gas is unclear. To approximate the photoelectric heating efficiency, we therefore used the analytical expression as obtained by Bakes \& Tielens (1994). Since this expression was obtained for a grain-size distribution with a lower limit of $3 \AA$, we scaled their result with a factor 0.2 to account for our $a_{\min }$ of $50 \AA$ (see their Fig. 8)

$$
\begin{aligned}
\hat{H}_{\mathrm{pe}}(r)= & 0.2 \times 10^{-24} \times \frac{3 \times 10^{-2}}{1+2 \times 10^{-4} G_{0} \sqrt{T(r)} / n_{\mathrm{e}}(r)} \\
& \times n\left(\mathrm{H}_{2}\right)(r)\left(f_{\mathrm{H}}(r)+2\right)
\end{aligned}
$$

in erg s $\mathrm{s}^{-1} \mathrm{~cm}^{-3}$, with $G_{0}=1$ the standard value for the intensity of the incident far-UV field in units of the Habing interstellar radiation field. This equation is obtained for a grain-size distribution $n_{\mathrm{d}}(a, r) \mathrm{d} a=A a^{-3.5} n_{\mathrm{H}} \mathrm{d} a$, with $A$ being $10^{-25.16}$. The electron density $n_{\mathrm{e}}(r)$ is calculated from the reaction $\mathrm{CO} \rightarrow \mathrm{O}+\mathrm{C} \rightarrow$ $\mathrm{O}+\mathrm{C}^{+}+\mathrm{e}^{-}$, which is known to be the main provider of electrons in the CSE. As demonstrated by Mamon et al. (1988) in their Fig. 6, the abundance of neutral $\mathrm{C}$ in the CSE is always less than the $\mathrm{CO}$ or $\mathrm{C}^{+}$abundance.

Equation (9) still has to be scaled with a factor $G_{0} \times$ $\exp \left(-\tau_{\mathrm{uv}}\right)$, where $\tau_{\mathrm{uv}}$ represents the optical depth of the shell against UV radiation as measured from the outer radius, i.e.

$H_{\mathrm{pe}}(r)=\hat{H}_{\mathrm{pe}}(r) \times G_{0} \times \exp \left(-\tau_{\mathrm{uv}}(r)\right)$.

The optical depth $\tau_{\mathrm{uv}}(r)$ is taken to be $1.8 A_{v}(r)$ (Hollenbach \& Tielens 1999). In case of an interstellar silicate-to-gas ratio the extinction $A_{v}=1.6 \times 10^{-22} \times N_{\mathrm{H}}$, with $N_{\mathrm{H}}$ the column number density of hydrogen. The constant is scaled to the appropriate dust-to-gas mass ratio of the shell, i.e. $A_{v}=1.6 \times 10^{-22} / 0.01 \times$ $\psi \times N_{\mathrm{H}}$.

\subsubsection{Heat exchange between dust and gas}

Since gas and dust have a different temperature, heat can be exchanged between the two species. This process can heat/cool the gas considerably, but has almost no effect on the dust temperature. The heating/cooling rate per unit volume in units of erg s $\mathrm{cm}^{-1}$ may be written as (Burke \& Hollenbach 1983)

$$
\begin{aligned}
& H_{\Delta T}(r)=\int n_{\mathrm{H}}(r) n_{\mathrm{d}}(a, r) \sigma_{\mathrm{d}}(a) \sqrt{\frac{8 k T(r)}{\pi m_{\mathrm{H}}}} \overline{\alpha_{T}(r)} \\
& \quad \times 2 k\left[T_{\mathrm{d}}(r)-T(r)\right] \mathrm{d} a \\
& =4 \pi k \sqrt{\frac{8 k}{\pi m_{\mathrm{H}}}} \times A(r) \times n^{2}\left(\mathrm{H}_{2}\right)(r)\left(f_{\mathrm{H}}(r)+2\right)^{2} \\
& \overline{\alpha_{T}}(r) \sqrt{T(r)}\left[T_{\mathrm{d}}(r)-T(r)\right]\left(a_{\mathrm{min}}^{-0.5}-a_{\text {max }}^{-0.5}\right),
\end{aligned}
$$

with $T_{\mathrm{d}}(r)$ the dust temperature and $\overline{\alpha_{T}}(r)$ the average accommodation coefficient (Burke \& Hollenbach 1983; Groenewegen 1994)

$\overline{\alpha_{T}}(r)=0.35 \exp \left(-\sqrt{\left(T_{\mathrm{d}}(r)+T(r)\right) / 500}\right)+0.1$.
Since we do not solve the radiative transfer in the dust continuum, the dust temperature as function of the grain-size $T_{\mathrm{d}}(a, r)$ is unknown. Instead, the dust temperature is assumed to vary as (Olofsson in Habing \& Olofsson 2003)

$T_{\mathrm{d}}(r)=T_{\star}\left(R_{\star} / 2 r\right)^{2 /(4+s)}$,

with $T_{\star}$ and $R_{\star}$ the temperature and radius of the central star. Observational data suggest that $s \approx 1$ (Olofsson in Habing \& Olofsson 2003).

\subsubsection{Heating by cosmic rays}

The heating rate per unit volume by cosmic rays is calculated by Goldsmith \& Langer (1978), and given by

$H_{\mathrm{cr}}(r)=6.4 \times 10^{-28} \times n\left(\mathrm{H}_{2}\right)(r)\left(1+f_{\mathrm{H}}(r) / 2\right)\left(1+4 f_{\mathrm{He}}(r)\right)$

in units of $\mathrm{erg} \mathrm{s}^{-1} \mathrm{~cm}^{-3}$. The uncertainty in the numerical factor is about a factor of two.

\subsubsection{Cooling by rotational excitation of $\mathrm{H}_{2} \mathrm{O}$}

Because the typical temperatures in the CSE around evolved stars are low, radiative cooling mechanisms involving the collisional excitation of the rotational levels of abundant molecules form the major sinks of energy. $\mathrm{H}_{2} \mathrm{O}$ (this section) and $\mathrm{CO}$ (next section) with their high dissociation energy and substantial dipole moment are very important in this respect.

It is beyond the scope of this paper to calculate the population of the many $\mathrm{H}_{2} \mathrm{O}$ rotational levels in a self-consistent manner. Instead, we will follow GS76 in approximating the structure of a $\mathrm{H}_{2} \mathrm{O}$ molecule by a three-level system: two rotational levels in the ground electronic vibrational state $v=0$ at energy $\sim k T$ and one rotational level at $v=1$. Assuming that all of the pure rotational transitions in the ground vibrational state have the same excitation temperature $T_{\text {exc }}$, the heat loss rate per unit volume per unit time is given by the difference between the collisional rate for excitation and de-excitation

$$
\begin{aligned}
C_{\mathrm{H}_{2} \mathrm{O}, \mathrm{rot}} & (r)=n\left(\mathrm{H}_{2} \mathrm{O}\right)(r) \times h v_{2,1}(r) \times\left[n(\mathrm{H})(r)\langle\sigma v\rangle_{\left(\mathrm{H}-\mathrm{H}_{2} \mathrm{O}\right)}\right. \\
+ & \left.n\left(\mathrm{H}_{2}\right)(r)\langle\sigma v\rangle_{\left(\mathrm{H}_{2}-\mathrm{H}_{2} \mathrm{O}\right)}+n(\mathrm{He})(r)\langle\sigma v\rangle_{\left(\mathrm{He}-\mathrm{H}_{2} \mathrm{O}\right)}\right] \\
\times & {\left[\exp \left(-h v_{2,1}(r) / k T(r)\right)-\exp \left(-h v_{2,1}(r) / k T_{\mathrm{exc}}(r)\right)\right] }
\end{aligned}
$$

with $v_{2,1}(r)$ the transition frequency $\left(=2.6 \times 10^{11} T_{\text {exc }}^{0.5}(r) \mathrm{s}^{-1}\right)$, and $\langle\sigma v\rangle(r)$ the inelastic collision rate constant. Quite often the $\mathrm{He}-\mathrm{H}_{2} \mathrm{O}$ inelastic collisional rate constant is assumed to be a factor $\sqrt{2}$ lower than the $\mathrm{H}_{2}-\mathrm{H}_{2} \mathrm{O}$ rate constant due to the difference in mass (e.g. Groenewegen 1994). However, as demonstrated by Phillips et al. (1996), excitation by para- $\mathrm{H}_{2}$ is not too different from excitation by $\mathrm{He}$ atoms at excitation temperatures from 20 to $140 \mathrm{~K}$, with most rates being within a factor 1-3 larger, but excitation by ortho- $\mathrm{H}_{2}$ is significantly different, with some rates an order of magnitude larger than rates for excitation by He atoms. From Tables 6 and 7 in Phillips et al. (1996), we use as mean values for the ratio of the inelastic collision rates

$$
\begin{aligned}
& \left|\frac{\langle\sigma v\rangle_{\left(\mathrm{H}_{2}(j=0)-\mathrm{H}_{2} \mathrm{O}_{\text {para }}\right)}}{\langle\sigma v\rangle_{\left(\mathrm{He}-\mathrm{H}_{2} \mathrm{O}_{\text {para }}\right.}}\right| \approx 1.7\left|\frac{\langle\sigma v\rangle_{\mathrm{H}_{2}(j=0)-\mathrm{H}_{2} \mathrm{O}_{\text {ortho }}}}{\langle\sigma v\rangle_{\left(\mathrm{He}-\mathrm{H}_{2} \mathrm{O}_{\text {ortho }}\right)}}\right| \approx 1.6 \\
& \left|\frac{\langle\sigma v\rangle_{\mathrm{H}_{2}(j=1)-\mathrm{H}_{2} \mathrm{O}_{\text {para }}}}{\langle\sigma v\rangle_{\left(\mathrm{He}-\mathrm{H}_{2} \mathrm{O}_{\text {para }}\right)}}\right| \approx 7.7\left|\frac{\langle\sigma v\rangle_{\mathrm{H}_{2}(j=1)-\mathrm{H}_{2} \mathrm{O}_{\text {ortho }}}}{\langle\sigma v\rangle_{\left(\mathrm{He}-\mathrm{H}_{2} \mathrm{O}_{\text {ortho }}\right)}}\right| \approx 7.8 .
\end{aligned}
$$

Theoretical rate constants among the lowest 45 para and 45 ortho rotational levels of water in collisions with $\mathrm{He}$ atoms have been 
calculated by Green et al. (1993). From their Tables 2 and 3, we derive

$$
\begin{aligned}
\langle\sigma v\rangle_{\left(\mathrm{He}-\mathrm{H}_{2} \mathrm{O}_{\text {para }}\right)} & \approx 0.21 \times \sqrt{T(r)} \times 10^{-11} \mathrm{~cm}^{3} \mathrm{~s}^{-1} \\
\langle\sigma v\rangle_{\left(\mathrm{He}-\mathrm{H}_{2} \mathrm{O}_{\text {ortho }}\right)} & \approx 0.13 \times \sqrt{T(r)} \times 10^{-11} \mathrm{~cm}^{3} \mathrm{~s}^{-1} .
\end{aligned}
$$

The $\mathrm{H}-\mathrm{H}_{2} \mathrm{O}$ inelastic rate constant is assumed to be a factor 1.16 larger than the $\mathrm{He}-\mathrm{H}_{2} \mathrm{O}$ constant (this value takes both the smaller mass and the smaller cross section of $\mathrm{H}$ into account). With an ortho-to-para ratio of 3:1 for both $\mathrm{H}_{2}$ and $\mathrm{H}_{2} \mathrm{O}$, we obtain as cooling rate for rotational excitation of $\mathrm{H}_{2} \mathrm{O}$

$$
\begin{aligned}
& C_{\mathrm{H}_{2} \mathrm{O}, \text { rot }}(r)=n\left(\mathrm{H}_{2}\right)(r) n\left(\mathrm{H}_{2} \mathrm{O}\right)(r) h v_{2,1}(r)\left[0.21 \times 10^{-11}\right. \\
& \quad \times \sqrt{T(r)}] \times\left[0.83 f_{\mathrm{H}}(r)+0.715 f_{\mathrm{He}}(r)\left\{f_{\mathrm{H}}(r)+2\right\}+4.5\right] \\
& \quad \times\left[\exp \left\{-h v_{2,1}(r) / k T(r)\right\}-\exp \left\{-h v_{2,1}(r) / k T_{\mathrm{exc}}(r)\right\}\right] .
\end{aligned}
$$

The excitation temperature $T_{\text {exc }}(r)$ can be calculated from the rate equations, using an escape probability formalism to describe the radiative transfer in the line; see Eq. (11) in J94.

The water molecules in the CSE are photodissociated by interstellar ultraviolet photons with $\lambda \approx 1650 \AA$. In particular, the photochemical chain is given by $\mathrm{H}_{2} \mathrm{O} \rightarrow \mathrm{OH}+\mathrm{H} \rightarrow \mathrm{O}+\mathrm{H}+\mathrm{H}$ (GS76; Huggins \& Glassgold 1982; Netzer \& Knapp 1987). This photodissociation of $\mathrm{H}_{2} \mathrm{O}$ is a significant source of $\mathrm{OH}$ molecules in the outer envelope. Since no functional form exists for the distribution of water in the CSE - and the implementation of a chemical reaction network programme is planned for the future - we assume a similar description for the $\mathrm{H}_{2} \mathrm{O}$ abundance profile as derived by Mamon et al. (1988) for $\mathrm{CO}$ (see next section, Eq. (22)). In this functional form, we have to provide the position $r_{1 / 2}^{\left(\mathrm{H}_{2} \mathrm{O}\right)}$ where the abundance of water relative to $\mathrm{H}_{2}$ has decreased by a factor of two relative to the value at the stellar surface. This value is set by (Groenewegen 1994)

$$
\begin{aligned}
r_{1 / 2}^{\left(\mathrm{H}_{2} \mathrm{O}\right)}= & 35\left(\overline{\dot{M}} / 10^{-5} M_{\odot} \mathrm{yr}^{-1}\right)^{0.7} \\
& \times\left(v(r) / 15 \mathrm{~km} \mathrm{~s}^{-1}\right)^{-0.4} 10^{15} \mathrm{~cm},
\end{aligned}
$$

where (in case of variable mass loss) $\overline{\dot{M}}$ is taken as the mean of $\dot{M}(r)$. This expression is derived from the results of Netzer \& Knapp (1987), who determined the $\mathrm{OH}$ peak number densities in the CSEs around evolved stars. The thus assumed $\mathrm{H}_{2} \mathrm{O}$ distribution is similar to the results of Nejad \& Millar (1988, see their Fig. 1).

\subsubsection{Cooling by rotational excitation of $\mathrm{CO}$}

Justtanont et al. (1994) included CO cooling in a similar way as $\mathrm{H}_{2} \mathrm{O}$ rotational cooling. The $\mathrm{H}-\mathrm{CO}$ inelastic rate constant is assumed to be a factor 1.16 larger than the He-CO inelastic rate constant due to the difference in mass (velocity) and cross section. Using (Schinke et al. 1985)

$\langle\sigma v\rangle_{\left(\mathrm{CO}-\mathrm{H}_{2}\right)} \approx 4 \times 10^{-12} \times \sqrt{T(r)} \mathrm{cm}^{3} \mathrm{~s}^{-1}$,

and

$\langle\sigma v\rangle_{(\mathrm{CO}-\mathrm{He})} \approx 4.5 \times 10^{-13} \times \sqrt{T(r)} \mathrm{cm}^{3} \mathrm{~s}^{-1}$,

(McKee et al. 1982), we obtain

$$
\begin{aligned}
& C_{\mathrm{CO}, \text { rot }}(r)=n\left(\mathrm{H}_{2}\right)(r) \times n(\mathrm{CO})(r) \times h v_{2,1}(r) \\
& \quad \times\left[4.5 \times 10^{-13} \times \sqrt{T(r)}\right] \\
& \quad \times\left[1.16 f_{\mathrm{H}}(r)+f_{\mathrm{He}}(r)\left\{f_{\mathrm{H}}(r)+2\right\}+10\right] \\
& \quad \times\left[\exp \left\{-h v_{2,1}(r) / k T(r)\right\}-\exp \left\{-h v_{2,1}(r) / k T_{x}(r)\right\}\right],
\end{aligned}
$$

with $v_{2,1}(r)=4.89 \times 10^{10} T_{x}^{0.5}(r) \mathrm{s}^{-1}$, and $T_{x}(r)$ the excitation temperature for $\mathrm{CO}$ molecules defined in a similar manner as $\mathrm{H}_{2} \mathrm{O}$ (see previous section).

An important quantity is the $\mathrm{CO}$ abundance in the photosphere, which is determined by the carbon and oxygen supply. During the first dredge-up on the red giant branch the carbon abundance is depleted to roughly two-thirds of the main sequence value while the oxygen abundance remains almost unchanged. On the AGB, carbon may be added to the envelope due to the third dredge-up. Typical $\mathrm{CO}$ and $\mathrm{H}_{2} \mathrm{O}$ abundances at the base of the CSE are $f_{\mathrm{CO}}\left(R_{\star}\right)=n(\mathrm{CO})\left(R_{\star}\right) / n\left(\mathrm{H}_{2}\left(R_{\star}\right)\right)=5.3 \times$ $10^{-4}$ and $f_{\mathrm{H}_{2} \mathrm{O}}\left(R_{\star}\right)=n\left(\mathrm{H}_{2} \mathrm{O}\right)\left(R_{\star}\right) / n\left(\mathrm{H}_{2}\left(R_{\star}\right)\right)=1.2 \times 10^{-3}$ when the star arrives on the AGB. If the $\mathrm{C} / \mathrm{O}$ ratio in an AGB star has increased to 0.7 , the abundances changes to $1.2 \times 10^{-3}$ and $5.1 \times 10^{-4}$ for $\mathrm{CO}$ and $\mathrm{H}_{2} \mathrm{O}$ respectively (Groenewegen 1994). Knapp \& Morris (1985) arrived at a value of $f_{\mathrm{CO}}\left(R_{\star}\right)=3 \times$ $10^{-4}$ for O-rich giants, $6 \times 10^{-4}$ for S-type stars, and $8 \times 10^{-4}$ for C-rich giants; Zuckerman \& Dyck (1986) used $5 \times 10^{-4}$ for O-rich and $1 \times 10^{-3}$ for S- and C-rich giants. This uncertainty in $f_{\mathrm{CO}}$ translates in an uncertainty on the derived mass-loss rate. In our code the carbon and oxygen abundances at the stellar radius $R_{\star}$ are free input parameters, and can be adjusted according to the evolutionary state of the target under study. If no information can be found on the carbon and oxygen abundances in the outer atmosphere of the target, we opt to use the cosmic abundance values of Anders \& Grevesse (1989).

CO will be dissociated by the interstellar UV field, and its abundance will decrease outward. Mamon et al. (1988) have investigated this effect on the $\mathrm{CO}$ abundance distribution. They obtained that the $\mathrm{CO}$ abundance $f_{(\mathrm{CO})}(r)$, relative to the value at the photosphere $f_{(\mathrm{CO})}\left(R_{\star}\right)$, can be approximated by

$f_{(\mathrm{CO})}(r)=f_{(\mathrm{CO})}\left(R_{\star}\right) \exp \left[-\ln 2\left(r / r_{1 / 2}^{(\mathrm{CO})}\right)^{\alpha}\right]$,

where $r_{1 / 2}^{(\mathrm{CO})}$ denotes the position where the number density has decreased by a factor of two. The values for $r_{1 / 2}^{(\mathrm{CO})}$ and $\alpha$ depend on the gas velocity and mass loss rate and are tabulated by Mamon et al. (1988).

\subsubsection{Cooling by vibrational excitation of $\mathrm{H}_{2}$}

Cooling by vibrational excitation of $\mathrm{H}_{2}$ molecules is simpler to calculate than for $\mathrm{H}_{2} \mathrm{O}$. The reasons are: (1) The vibrational energy level spacing is large $(\sim 0.6 \mathrm{eV})$. Hence only collisional excitation of the first excited vibrational state needs to be considered. (2) Since $\mathrm{H}_{2}$ is dipole inactive, resulting in small Einstein $A$-values, the lines are optically thin even in the dense part of the CSE. (3) $\mathrm{H}_{2}$ is in LTE through a large part of the envelope (GS76, J94). The description of the heat loss per unit volume is taken from GS76, and can be summarised as

$C_{\mathrm{H}_{2}}=A_{1,0} h v_{1.0} n_{1}(r)$,

where $A_{1,0} \approx 3 \times 10^{-7} \mathrm{~s}^{-1}$ is the spontaneous emission rate from the first vibrational state, $h v_{1,0} \approx 0.6 \mathrm{eV}$ is the energy of the emitted photon, and $n_{1}=n_{\mathrm{H}_{2}}(v=1)$ the number density of vibrationally excited $\mathrm{H}_{2}$ molecules. The number density $n_{1}$ of vibrationally excited $\mathrm{H}_{2}$ by collisions with $\mathrm{H}_{2}$ and $\mathrm{H}$ atoms, can be written as

$n_{1}(r)=n\left(\mathrm{H}_{2}\right)(r) \frac{\alpha_{n}(r) \times \exp \left(-h v_{1,0} / k T(r)\right)}{\alpha_{n}(r) \times\left[1+\exp \left(-h v_{1,0} / k T(r)\right)\right]+A_{1,0}}$

with

$\alpha_{n}(r)=n(\mathrm{H})(r)\left\langle\sigma^{*} v_{t h}\right\rangle_{\left(\mathrm{H}-\mathrm{H}_{2}\right)}+n\left(\mathrm{H}_{2}\right)(r)\left\langle\sigma^{*} v_{t h}\right\rangle_{\left(\mathrm{H}_{2}-\mathrm{H}_{2}\right)}$ 
where $\left\langle\sigma^{*} v_{t h}\right\rangle_{\left(\mathrm{H}-\mathrm{H}_{2}\right)}$ and $\left\langle\sigma^{*} v_{t h}\right\rangle_{\left(\mathrm{H}_{2}-\mathrm{H}_{2}\right)}$ are the collisional deexcitation rate constants.

Theoretical values for $\left\langle\sigma^{*} v_{t h}\right\rangle_{\left(\mathrm{H}-\mathrm{H}_{2}\right)}$ are approximated by (Hollenbach \& McKee 1979)

$$
\begin{aligned}
\left\langle\sigma^{*} v_{t h}\right\rangle_{\left(\mathrm{H}-\mathrm{H}_{2}\right)}= & 1.0 \times 10^{-12} T(r)^{1 / 2} \\
& \times \exp [-(1000 / T(r))] \mathrm{cm}^{3} \mathrm{~s}^{-1},
\end{aligned}
$$

and for $\left\langle\sigma^{*} v_{t h}\right\rangle_{\left(\mathrm{H}_{2}-\mathrm{H}_{2}\right)}$ (Hollenbach \& McKee 1989)

$$
\begin{aligned}
\left\langle\sigma^{*} v_{t h}\right\rangle_{\left(\mathrm{H}_{2}-\mathrm{H}_{2}\right)}= & 1.4 \times 10^{-12} T(r)^{1 / 2} \\
& \times \exp \{-[18100 /(T(r)+1200)]\} \mathrm{cm}^{3} \mathrm{~s}^{-1} .
\end{aligned}
$$

\subsection{Treatment of the boundary conditions}

The differential equations governing the temperature and velocity structure (Eqs. (2) and (7)) are solved simultaneously. For the central star, we use a blackbody with a user-defined temperature $T_{\star}$. As inner boundary for the velocity structure, we assume that the flow velocity of the gas is equal to the local sound velocity, $v_{s}$, at the radius of the CSE, $R_{\text {inner }}$, where the dust condenses. Since many questions still exist on the complex structure between $R_{\star}$ and $R_{\text {inner }}$ we assume that the temperature in this regime is described by a power law

$T(r)=T_{\star}\left(\frac{R_{\star}}{r}\right)^{\zeta}$

with $\zeta \approx 0.5$, which holds when both emission and absorption are optically thin. The velocity law can be approximated by the classical $\beta$-law

$v(r) \simeq v_{\infty}\left(1-\frac{R_{0}}{r}\right)^{\beta}$

with

$R_{0}=R_{\star}\left\{1-\left(\frac{v_{0}}{v_{\infty}}\right)^{1 / \beta}\right\}$

with $v_{0}$ the velocity at the photosphere, which can be calculated directly from $v_{s}$, and $\beta$ being $1 / 2$, a typical value in case of winds of cool stars (Schutte \& Tielens 1989). Since we are studying low excitation rotational $\mathrm{CO}$ line profiles being formed quite far in the envelope, the exact value of $\epsilon$ and $\beta$ does not influence the calculated line profiles. The outer radius is determined by the $\mathrm{CO}$ abundance, and is set at the radius where the $\mathrm{CO}$ abundance $f_{\mathrm{CO}}(r)$ drops to $1 \%$ of its value at the photosphere. Adopting a value for the gas mass-loss rate, the dustto-gas ratio is varied until the observed terminal velocity is obtained. Since $\Gamma(r) \propto \psi \cdot Q_{\lambda}(a)$ (Eq. (5)), the derived dust-to-gas ratio $\psi$ should be interpreted in relation to the used tabulated extinction efficiencies $Q_{\lambda}(a)$ derived by Justtanont \& Tielens (1992) for silicate grains.

The sudden onset of some heating or cooling terms, yields a change in going from a non-stiff to a stiff set of differential equations (or vice versa), which a standard Runge-Kutta method is not able to handle. We therefore chose to use the DLSODA package as provided by ODEPACK ${ }^{2}$. Going from the inner boundary to the outer boundary, this solver automatically selects between non-stiff and stiff methods. It uses Adams methods (predictor-corrector) in the non-stiff case, and Backward Differentiation Formula methods (the Gear methods) in the stiff case. We require an absolute accuracy of $1 \mathrm{~K}$ in temperature and $100 \mathrm{~cm} \mathrm{~s}^{-1}$ in velocity, i.e. the estimated local error in the parameter is less than the above-mentioned accuracy.

\footnotetext{
${ }^{2}$ http://www.netlib.org/odepack/
}

Table 1. Parameters of GX Mon as derived by J94.

\begin{tabular}{ll}
\hline \hline Parameter & Value \\
\hline$T_{\star}$ & $2500 \mathrm{~K}$ \\
$R_{\star}$ & $4 \times 10^{13} \mathrm{~cm}$ \\
$M_{\star}$ & $1 M_{\odot}$ \\
$R_{\text {inner }}$ & $6.25 R_{\star}$ \\
$\dot{M}$ & $7.2 \times 10^{-6} M_{\odot} \mathrm{yr}^{-1}$ \\
$v_{\text {stoch }}$ & $1 \mathrm{~km} \mathrm{~s}^{-1}$ \\
Distance & $740 \mathrm{pc}^{-1}$ \\
$v_{\infty}$ & $19 \mathrm{~km} \mathrm{~s}^{-1}$ \\
\hline
\end{tabular}

\subsection{Variable mass loss}

It is beyond the scope of this project to implement a full hydrodynamical code to predict variabilities in the wind structure. We simulate the effect of various types of variable mass loss, as e.g. the superwind phase or "quasi-periodic variations" discussed in Sect. 1, in an empirical manner by adapting the mass-loss rate as a function of radius, i.e. $\dot{M}(r)$.

In order to incorporate a variable mass loss, we calculate for each radial point $r$ the expected temperature and velocity as if resulting from a constant mass outflow with the corresponding mass loss rate. In practise this means that we solve Eqs. (2) and (7) from the inner edge $R_{\text {inner }}$ up to $r$ with a fixed $\dot{M}$ chosen to correspond to $\dot{M}(r)$. In this way we assume that the main influence on the temperature is set by the expansion of the flow and the local heating and cooling terms. In this approach the influence of the neighbouring shells, which may correspond to different mass-loss rates, is neglected.

If the mass-loss behaviour is changed, the drift velocities will be affected. This in turn causes a change in the flow velocity $v(r)$ of the gas. This feedback is self-consistently accounted for in the calculations of the heating and cooling rates. However, we do not take these modified velocities into account when solving the radiative transfer equation, because our theoretical code solves the radiative transfer using the comoving frame (CMF) method as developed by Mihalas et al. (1975, see next section) which demands that the velocity should increase continuously with radius. In case of variable mass loss, we use the velocity structure as inferred from the case with constant $\dot{M}$. For instance, in the test case of GX Mon described in Sect. 2.5, the velocity structure as in the absence of a superwind is used.

Several options are built in the code to define $\dot{M}(r)$, being constant, a heavy-side function, a block function, a picket-fence function or analogous functions with smoother derivatives.

\subsection{Discussion}

To check the new input of heating and cooling rates, and new numerical routines we simulated the results as obtained by $\mathrm{J} 94$ for the Mira-type star GX Mon. Parameters as derived by J94 are listed in Table 1. Our final temperature and velocity structure are displayed in Fig. 1 in the lower right and upper right panel respectively, and should be compared with Fig. 1 in J94.

Inspecting the gas and drift velocity structure shows quite a difference between both results. The reason for this difference is a mistake in the implementation of the results of the ordinary differential equation (ODE) operator (being a RungeKutta method with adaptive stepsize control) in the version of J94. J94 erroneously assigned the computed parameters being the velocity $v(r 1)$, temperature $T(r 1)$ and the respective derivatives $\mathrm{d} v(r 1) / \mathrm{d} r$ and $\mathrm{d} T(r 1) / \mathrm{d} r$ at radius point $r 1$ also to the next radius point $r 2$ in the grid, which input was then used to calculate 


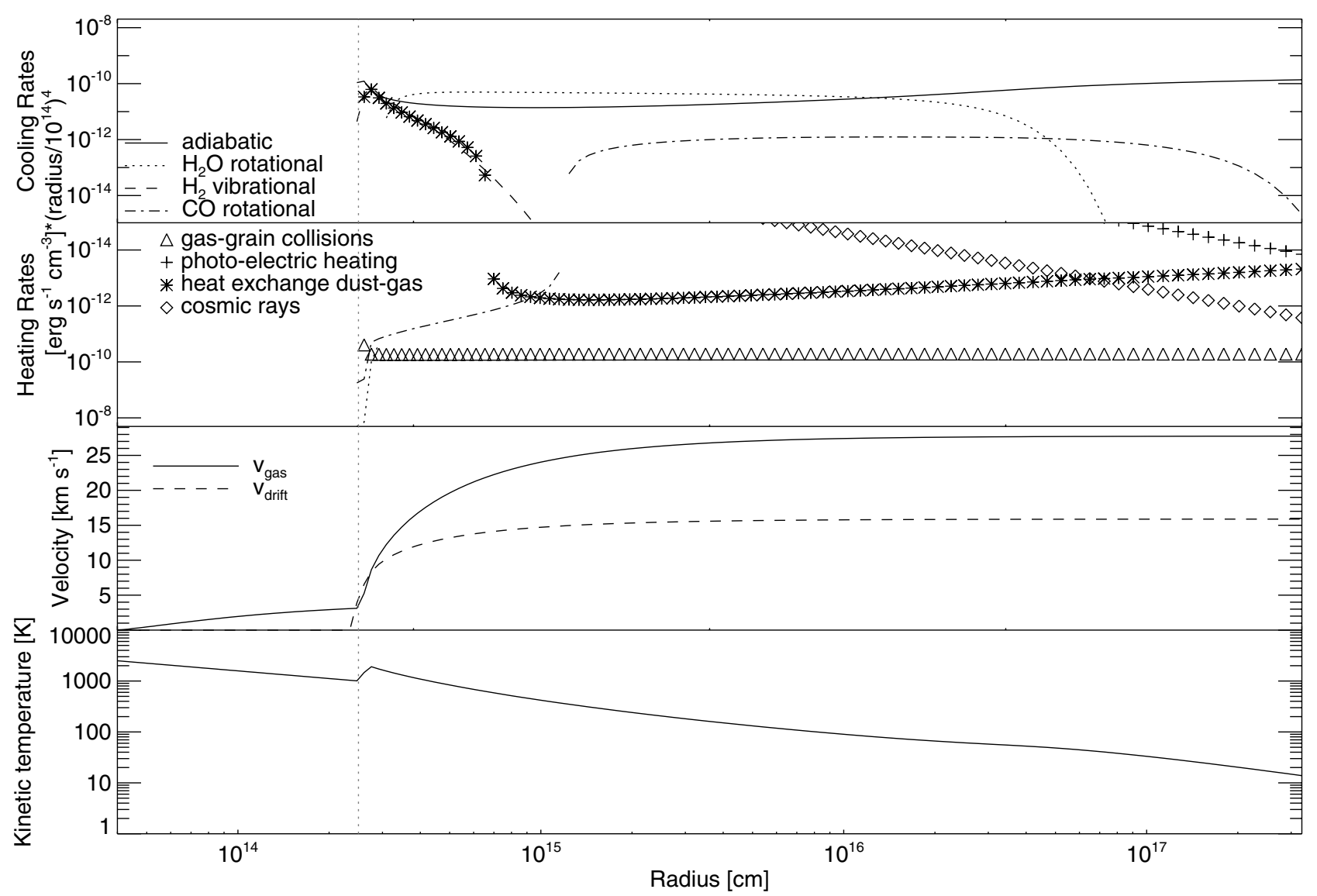

Fig. 1. The velocity structure and gas kinetic temperature from calculating the energy sources and sinks in the CSE of GX Mon. The start of the dusty envelope, $R_{\text {inner }}$, is indicated by the dotted line. The drift velocity is displayed for the maximum grain-size, $a=0.25 \mu \mathrm{m}$. Note that for grains with radius smaller than the wavelength, $Q \propto a$; hence $v_{\text {drift }} \propto a^{0.5}$. First panel: cooling rates; second panel: heating rates; third panel: velocity structure; fourth panel: gas kinetic temperature.

the parameters at the newly derived radius point $r 2^{\prime}$, computed using adaptive stepsize control. This inaccuracy yielded in case of GX Mon a terminal velocity being $\sim 8 \mathrm{~km} \mathrm{~s}^{-1}$ too low and a terminal drift velocity being $\sim 5 \mathrm{~km} \mathrm{~s}^{-1}$ too low. Moreover, our improved treatment of the drift velocity (Eq. (4), Sect. 2.1) lowers for GX Mon the terminal drift velocity by $0.5 \mathrm{~km} \mathrm{~s}^{-1}$ and the terminal flow velocity by $2 \mathrm{~km} \mathrm{~s}^{-1}$ compared to the approximation of $v_{\text {drift }}(a, r)=v_{K}(a, r)(\mathrm{J} 94)$.

That there is some difference between the derived temperature structures does not come as a surprise, since new heating terms are included and some of the ones already accounted for underwent major updates.

- Heat exchange between dust and gas and cosmic ray heating have been included, but they only have a minor contribution to the total heating rate.

- The gas-grain collisional heating rate as given by J94 is somewhat too low due to a numerical mistake in the implementation of the fractional abundance of atomic hydrogen $f_{\mathrm{H}}$ yielding too low a scaling factor $A$. Since this frictional heating is the main heating source over most of the envelope regime, our calculated $T(r)$ is in general higher than in J94.

- The main differences in the cooling rates come from the updates of the inelastic collisional rates involved in the cooling by rotational excitation of $\mathrm{H}_{2} \mathrm{O}$ and $\mathrm{CO}$, being lower than in $\mathrm{J} 94$.

As an illustration of the effects of variations in the mass-loss rate, we simulate a superwind phase with an enhanced mass loss of a factor of ten till $200 R_{\star}\left(=8 \times 10^{15} \mathrm{~cm}\right)$ compared to the parameters of the base-line model of GX Mon given in Table 1. We do not attempt to better explain the observed line profiles of GX Mon. In Sect. 4 we will present the results of a parameter study focusing on explaining the observations of VY CMa.

Figure 2 displays the temperature, velocity, heating and cooling terms for this simulation. As explained in Sect. 2.4, the velocity structure used in the radiative transfer calculations is not coupled to a change in mass-loss rate, but is kept as inferred from a case with constant $\dot{M}$. In the panel giving the velocity structure, the black lines represent the gas and drift velocity in case $\dot{M}=7.2 \times 10^{-5} M_{\odot} \mathrm{yr}^{-1}$ would have been used to calculate the velocity structure used in the radiative transfer calculations, the blue (grey) line is for $\dot{M}=7.2 \times 10^{-6} M_{\odot} \mathrm{yr}^{-1}$. An increase in $\dot{M}$ yields (1) a decrease of the drift velocity $\left(v_{\text {drift }} \propto \dot{M}^{-1 / 2}\right.$, Eq. (4)) since the coupling between dust and gas increases, and (2) a higher terminal velocity, since there is more dust to intercept the radiation and hence to accelerate the gas by transfer of momentum. Which constant $\dot{M}$ value to choose in this case can be debated, but as illustrated in Fig. 2, the difference between both $v(r)$-structures is small. A more correct gas velocity profile should display a decrease of the flow velocity from $200 R_{\star}(=8 \times$ $\left.10^{15} \mathrm{~cm}\right)$ on.

The change in viscous heating is not dominated by the decrease in drift velocity, but by the increase in dust and $\mathrm{H}_{2}$ particles, yielding a higher gas-grain collisional heating rate out to $200 R_{\star}$. Other energy rates are increased too, with the total influence being quite complex. In general, the temperature is higher 


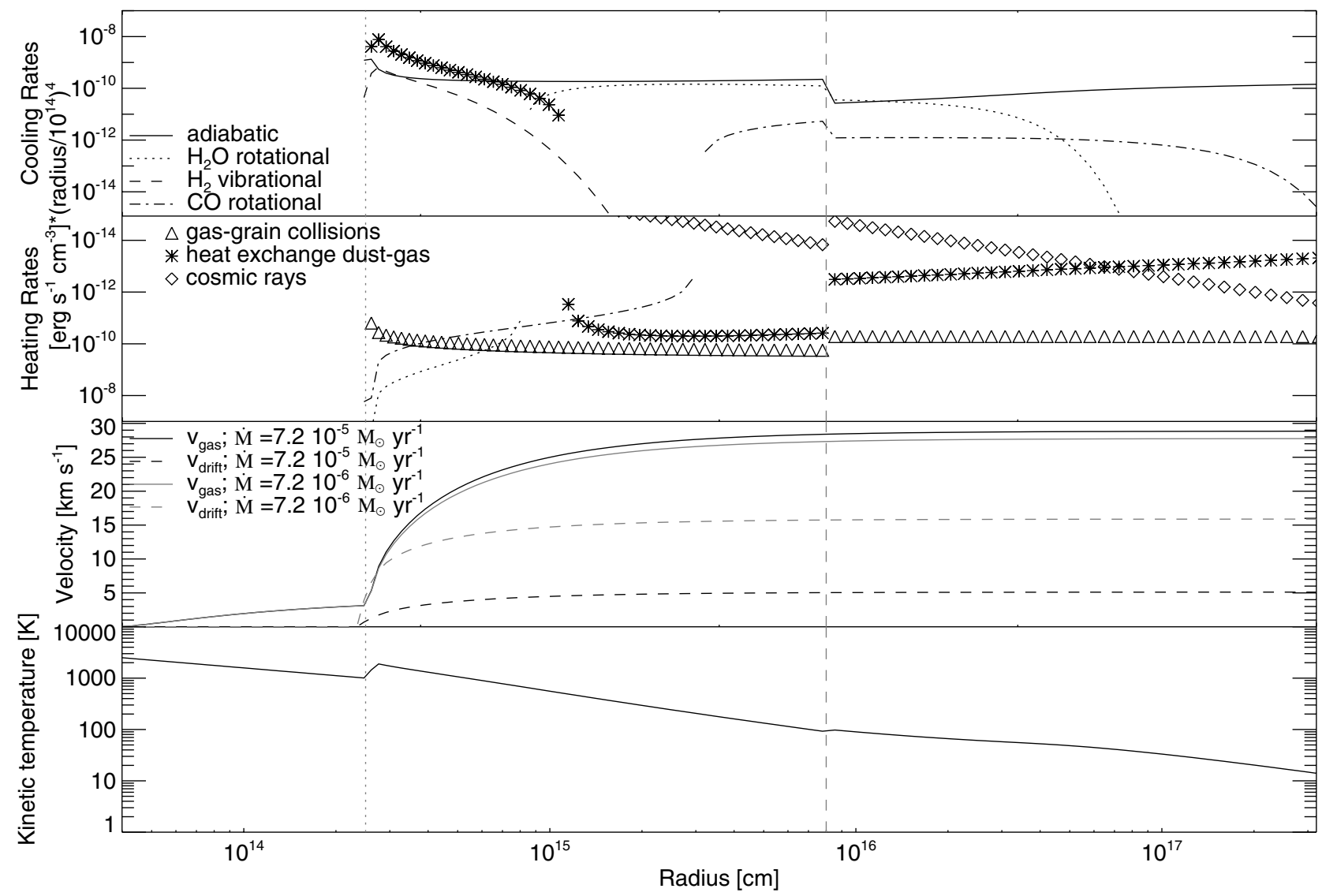

Fig. 2. Same as Fig. 1, but now with a superwind phase, i.e. with a mass-loss rate being a factor of 10 higher up to $200 R_{\star}$ (indicated by the dashed line). The velocity structure displayed in Fig. 1 is also shown in grey in the third panel. More details are given in the text.

till $200 R_{\star}$ w.r.t. the constant mass-loss case displayed in Fig. 1. The effects of this superwind phase on the line profiles are discussed in Sect. 3.3.

\section{Line radiative transfer}

Late type giants and supergiants have a ratio of stochastic to terminal velocity that may be in the order of magnitude of $\sim 0.5$ or even 1 (Che et al. 1983) yielding rather broad scattering zones and thus a highly non-local radiative transfer. Therefore the condition for using the Sobolev approximation (Sobolev 1947; Castor 1970) is not fulfilled anymore. Schönberg (1985) demonstrated that non-local scattering effects cause severe errors in the source function when using the Sobolev approximation (see also J94).

Moreover, as demonstrated by Schönberg (1988), part of the line profile of the low-excitation CO transitions is formed under non-LTE circumstances. The decoupling radius (i.e. that distance from the star where the excitation temperature in the lines is by ten percent smaller than the kinetic temperature) is a function of the specific line. The higher the excitation level, the smaller the decoupling radius. Clearly, the decoupling radius decreases with decreasing mass-loss rate. For a model with parameters almost identical to the ones listed in Table 1, Schönberg (1988) demonstrated that the excitation of the rotational ${ }^{12} \mathrm{CO}$ lines in the vibrational ground state is dominated by collisions in the mass-loss range $\dot{M} \gtrsim 3 \times 10^{-5} M_{\odot} \mathrm{yr}^{-1}$.

We therefore opt to calculate the $\mathrm{CO}$ rotational line profiles using a non-LTE radiative transfer code based on the multilevel approximate Newton-Raphson (ANR) operator as derived by Schönberg \& Hempe (1986). The ANR operator has been derived from the comoving frame equation of radiation transfer (Mihalas et al. 1975). The method is similar to the partial linearision approach. The ANR operator is a diagonal (therefore local) operator, and can be derived with only a slight increase in computational effort over that required to find the formal solution. When solving for multilevel line formation in expanding envelopes, Schönberg \& Hempe (1986) have shown that convergence was reached within 10-20 iterations for winds around cool giants. We note that although the method is proven to be stable, Hillier (1990) demonstrated that at very high optical depths the diagonal ANR operator may give rise to damped, depthcoupled oscillations, which may be due to the inability of the diagonal operator to correctly handle the diffusion approximation. He has shown that in these cases tridiagonal (or pentadiagonal) Newton-Raphson operators have a superior convergence rate.

A description of the radiative transfer equations can be found in Schönberg (1988). The calculations include excitation by collisions with $\mathrm{H}_{2}$ as well as scattering of infrared and microwave radiation emergent from warm dust particles, from the stellar photosphere and the cosmic background radiation. The implementation of the dust opacity was modified to include the grain opacity of silicates as computed in Justtanont \& Tielens (1992).

After solving the radiative transfer, line profiles for each transition are calculated by ray-tracing using formal integration (Schönberg 1988). Writing the calculated flux as $F_{v}$, the main beam temperature $T_{\mathrm{MB}}$ is calculated by (Schönberg 1988)

$T_{\mathrm{MB}}=F_{v} \frac{\pi D_{\mathrm{TEL}}^{2}}{8 k}$ 


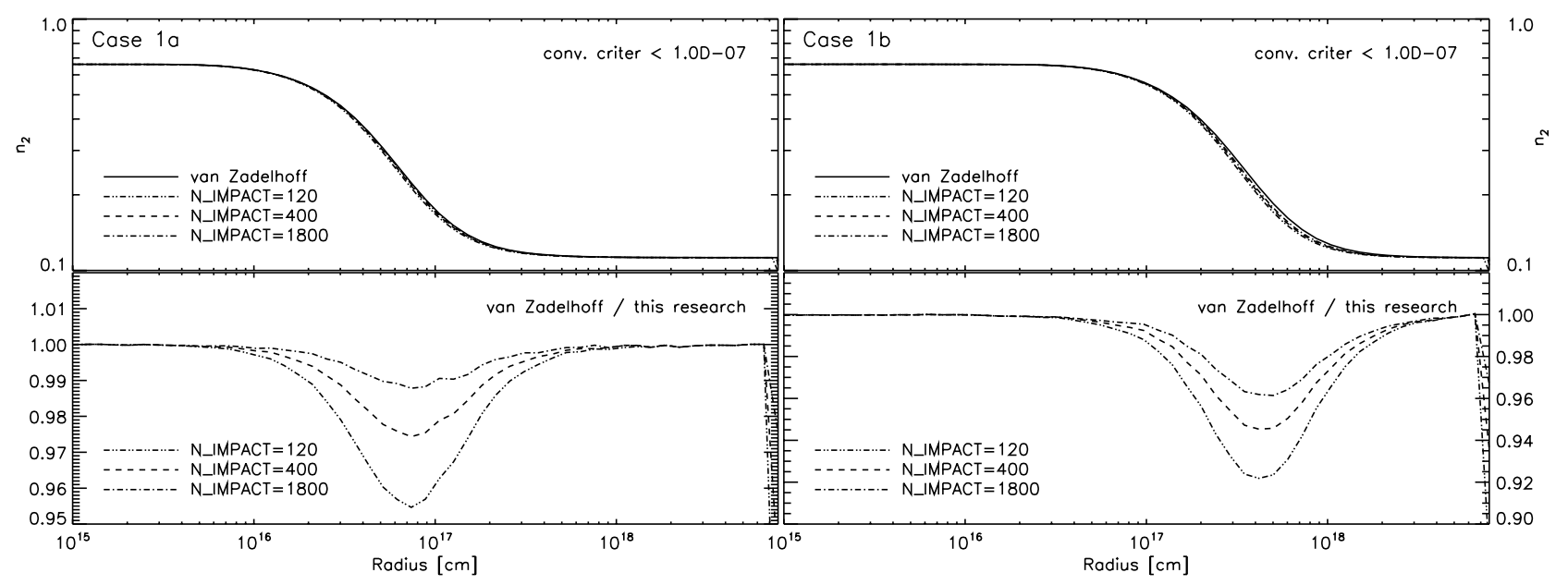

Fig. 3. Comparison of the benchmark case 1a (left) and $1 \mathrm{~b}($ right) as defined in van Zadelhoff et al. (2002) for different spatial resolutions. Upper panel: number density $n_{2}$ in the upper level of the fictive 2-level molecule as a function of radial distance from the star. The full line represents the mean of the number densities of all codes discussed by van Zadelhoff et al. (2002). Lower panel: ratio between their and our result. For increasing resolution the solutions converge. Dotted line: $\Delta R / R \approx 0.07$; dashed line: $\Delta R / R \approx 0.02$; dashed-dotted line $\Delta R / R \approx 0.005$.

with $D_{\mathrm{TEL}}$ the diameter of the antenna. Note that the line profile will not be symmetric since the opacity in the blue wing is produced at slightly larger $r$ than in the red wing. This kind of asymmetry in double-peaked (optically thin) line profiles will be stronger for resolved envelopes, and provides a useful diagnostic for obtaining the stochastic velocity (see Schönberg 1988).

\subsection{The molecular model}

The rate coefficients for collisions of $\mathrm{CO}$ with $\mathrm{H}_{2}$ at kinetic temperatures from 10 to $4000 \mathrm{~K}$ are taken from Larsson et al. (2002). In this work, rotational quantum numbers up to $J_{u}=40$ were taken into account. Data on the vibrational-rotational $v=1-0$ and the rotational transitions are from the HITRAN database (Rothman et al. 1987). A total of 62 levels and 120 transitions are treated, of which 31 levels are in the ground state of ${ }^{12} \mathrm{C}^{16} \mathrm{O}$, and 31 levels in the first vibrational state. Comparison with the CO line list of Goorvitch \& Chackerian (1994) shows that the used Einstein $A$-coefficients are in general good agreement; they are however systematically lower than the ones of Goorvitch \& Chackerian (1994), with a mean difference of a factor of 1.017 , a maximum of a factor 1.035 , and a minimum of a factor 1.005 .

\subsection{Benchmarking}

To test the accuracy, the new code has been benchmarked against existing radiative transfer codes. We used the study performed by van Zadelhoff et al. (2002), who compared a number of independent computer programs for radiative transfer in molecular rotational lines. None of the codes discussed in this comparison solves the radiative transfer in the comoving frame. While in the rest (inertial) frame the precision of a numerical code can be increased by using a second-order (or higher) integration of the transfer equation, in the comoving frame formalism it is written as two first-order differential equations yielding only first-order accuracy (Mihalas et al. 1975). In the case study of problems $1 \mathrm{a} / 1 \mathrm{~b}$ [having a simple power law density, constant temperature and a fictive 2-level molecule], the use of a first-order integration introduces errors of the order of $12 \%$ (van Zadelhoff et al. 2002). Hence, for a proper benchmarking of the code, the spatial resolution in the CMF should be increased to "mimic" a higher-order solution, i.e. to improve the accuracy.
This is illustrated in Fig. 3, for case 1a and 1b. It is clear that higher spatial resolution results in a better agreement with the other (non-CMF) codes, reaching a $1.2 \%$ level of accuracy for $\Delta R / R \approx 0.005$.

Case 2 of van Zadelhoff et al. (2002), describing an insideout collapsing envelope observed in rotational transitions of $\mathrm{HCO}^{+}$, has not been simulated since the CMF method is designed for a continuous increasing velocity, implying a bluewing condition stating that the highest frequency point in the local profile can intercept only continuum radiation from other points in the atmosphere.

\subsection{Effect of variable mass loss on the predicted line profiles of $\mathrm{CO}$}

For the specific case of GX Mon and the simulation of a superwind phase with the same parameters as in Sect. 2.4 the calculated low excitation $\mathrm{CO}$ line profiles are displayed in Fig. 4. The adopted beam sizes are those for the JCMT, and are indicated in the upper corner of each panel. Number densities, source functions $S_{v}$ and absorption coefficient $\kappa_{v}$ as a function of radius are plotted in Figs. 5 and 6.

A superwind phase increases the number densities of all the energy levels from $R_{\text {inner }}$ to $200 R_{\star}$, where the high mass-loss steps.

The sudden rise in temperature at the start of dust condensation gives a strong peak in the source function $S_{v}$ at $R_{\text {inner. The }}$ higher temperature in the superwind phase, results in (1) higher values of the source function up to $200 R_{\star}$, and lower values thereafter, and (2) a strong increase in the opacity up to $200 R_{\star}$ due to the higher number densities till the sudden decrease in mass loss rate introduces a sharp decrease in opacity.

The figures displaying $I(p) * p^{3}$ are instructive plots to illustrate where the lines mostly originate (see also Kemper et al. 2003). In case of a constant mass-loss rate, there is only one peak in $I(p) * p^{3}$, being closer to the star for higher excitation lines; for the superwind case, we see a second peak arising around $200 R_{\star}$, the end of the enhanced mass loss. A direct result from the enhancement in number densities at distances $\leq 200 R_{\star}$ is an increase in integrated intensity for the superwind case, especially for the higher excitational rotational lines which are formed closer in. Moreover, the sudden change in opacity 


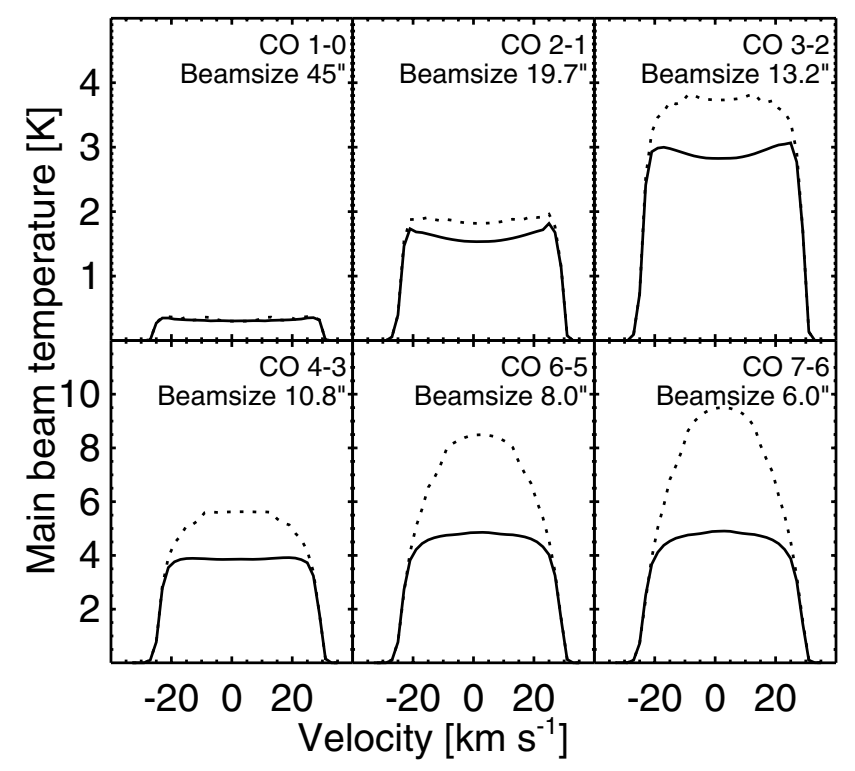

Fig. 4. CO rotational line profiles (a) full line: for GX Mon using $T(r)$ and $v(r)$ of Fig. 1, (b) dotted line: in case of a variable mass-loss rate as displayed in Fig. 2. Note the different ordinate axes between upper and lower panels.

(number density) at $200 R_{\star}$ gives rise to "bumps" in the line profiles, here with (projected) velocities around 10 and $15 \mathrm{~km} \mathrm{~s}^{-1}$.

Depending on the stellar input parameters, the abruptness of the variation in $\dot{M}$, and the place where (or time when) this change has taken place different kind of "composite" profiles (i.e. displaying "dips and bumps" in the line profile) are seen. This kind of composite profiles is also seen in observational data, with $\alpha$ Ori and VY CMa being examples in Kemper et al. (2003), and other targets in Winters et al. (2003). Note that the relative strength of the observed profiles of some stars, where this kind of "composite" profile is not visible, is also indicative for a variability in the mass-loss rate (see the discussion on WX Psc in Kemper et al. 2003).

The complex interplay between stellar and mass-loss parameters and some general trends linking the mass-loss rate to the observed line intensity will be described in a forthcoming paper.

\section{VY CMa}

To illustrate the diagnostic strength of the rotational $\mathrm{CO}$ profiles, we now apply our code to model the observed $\mathrm{CO}$ rotational line transitions emitted in the CSE around VY CMa. In Sect. 4.1, the basic parameters of this supergiant are summarised. The CO line profiles used in the analysis are described in Sect. 4.2. Sections 4.3 and 4.4 are devoted to the modelling of the CO profiles. We will demonstrate that it is possible to derive the mass-loss history of this target in great detail. Our results are compared to other studies in Sect. 4.5, and discussed in Sect. 4.6.

\subsection{Basic parameters of VYCMa}

VY CMa is a highly luminous, variable M2/3II supergiant. The star is heavily obscured, with only 1 percent of the total luminosity being detected at optical wavelengths (Le Sidaner \& Le Bertre 1996). The distance, luminosity, effective temperature and stellar radius have been estimated by several groups. These studies seem to converge on a distance of $1500 \mathrm{pc}$, a luminosity between $2-5 \times 10^{5} L_{\odot}$, and an effective temperature of $2800 \mathrm{~K}$
(Harwit et al. 2001; Le Sidaner \& Le Bertre 1996; Monnier et al. 1999). The current mass of VY CMa is estimated to be $15 M_{\odot}$ (Wittkowski et al. 1998). These values are adopted for the stellar parameters of VY CMa; they are summarised in Col. 2 in Table 2.

The $\mathrm{OH}, \mathrm{H}_{2} \mathrm{O}$, and $\mathrm{SiO}$ maser emission indicate expansion velocities between $\sim 30$ and $\sim 40 \mathrm{~km} \mathrm{~s}^{-1}$ (Reid \& Dickinson 1976; Snyder \& Buhl 1975; Reid \& Muhleman 1978). Modelling of $\mathrm{CO}$ rotational lines, $\mathrm{OH}$ maser emission and (near)-infrared data tracing the dust properties indicate values for the mass loss between $2.3 \times 10^{-5}$ and $4 \times 10^{-4} M_{\odot} \mathrm{yr}^{-1}$ (see Table 3 ). VY CMa's very high mass-loss rate fuels its optically thick circumstellar envelope and creates a rich infrared excess spectrum (see the 2.4-200 $\mu \mathrm{m}$ ISO spectrum in Harwit et al. 2001): metallic Fe and amorphous silicates dominate the infrared spectrum, with possible minor contributions from crystalline forsterite and crystalline enstatite.

\subsection{Observational data of VY CMa}

The rotational CO (2-1), (3-2), (6-5) and (7-6) line profiles have been observed by Kemper et al. (2003) with the JCMT and the CO (1-0) line by Nyman et al. (1992) with the SEST. The flux calibration accuracy for the JCMT data was estimated to be $10 \%$ for the CO (2-1) and (3-2) lines, and $~ 30 \%$ for the CO (6-5) and (7-6) lines since no reliable flux standards are available for these higher excitation lines. The $\mathrm{CO}(1-0)$ line has a main beam temperature of $0.06 \mathrm{~K}$, with an rms noise of $0.019 \mathrm{~K}$ (Nyman et al. 1992). As can be seen from Fig. 8, the profiles show a complex structure. Especially the different peaks present in the $\mathrm{CO}(2-1)$ and (3-2) profiles are indicative for variation $(s)$ in the mass-loss rate.

\subsection{A spherically symmetric envelope?}

There exist indications that the CSE surrounding VY CMa is not spherically symmetric. While some authors interpret the complex optical and infrared data in terms of an expanding disk or a bipolar outflow near the central object $\left(<0.2^{\prime \prime}\right.$; e.g. Herbig 1972; Bowers et al. 1983; Efstathiou \& Rowan-Robinson 1990), other data do not reveal indications for a disk-like or bipolar geometry (e.g. Monnier et al. 1999). Recently, "Hubble Space Telescope" in combination with ground-based infrared images revealed a complex structure of knots and filamentary arcs in the asymmetric reflection nebula several arcseconds across, around the obscured central star (Smith et al. 2001).

Nevertheless, we will assume spherical symmetry in modelling the molecular lines arising from the CSE. This is justified with the following arguments: (1) if our assumption of spherical symmetry is incorrect, this is more so for the innermost regions, where there are indications of a disk with extent $\lesssim 0.2^{\prime \prime}\left(\sim 30 R_{\star}\right)$. We are however aiming at a reconstruction of the mass-loss history from low-excitation CO lines, which are formed further out, and trace a region up to a few thousand stellar radii. (2) If the inhomogeneities are due to blobs of which the dimensions are modest compared to the total extent of the wind and which are more or less homogeneously distributed in the wind, the use of a spherically symmetric model gives us the average of the mass loss over all directions. In that way, our spherical model gives us an idea on the general mass-loss history of VY CMa. (3) Since the beam profiles of the instruments used to observe the $\mathrm{CO}$ lines are quite large (with a half power beam width (HPBW) of $45^{\prime \prime}$ in the $\mathrm{CO}(1-0)$ line, $19.7^{\prime \prime}$ in the $\mathrm{CO}(2-1), 13.2^{\prime \prime}$ in the $\mathrm{CO}(3-2)$, 


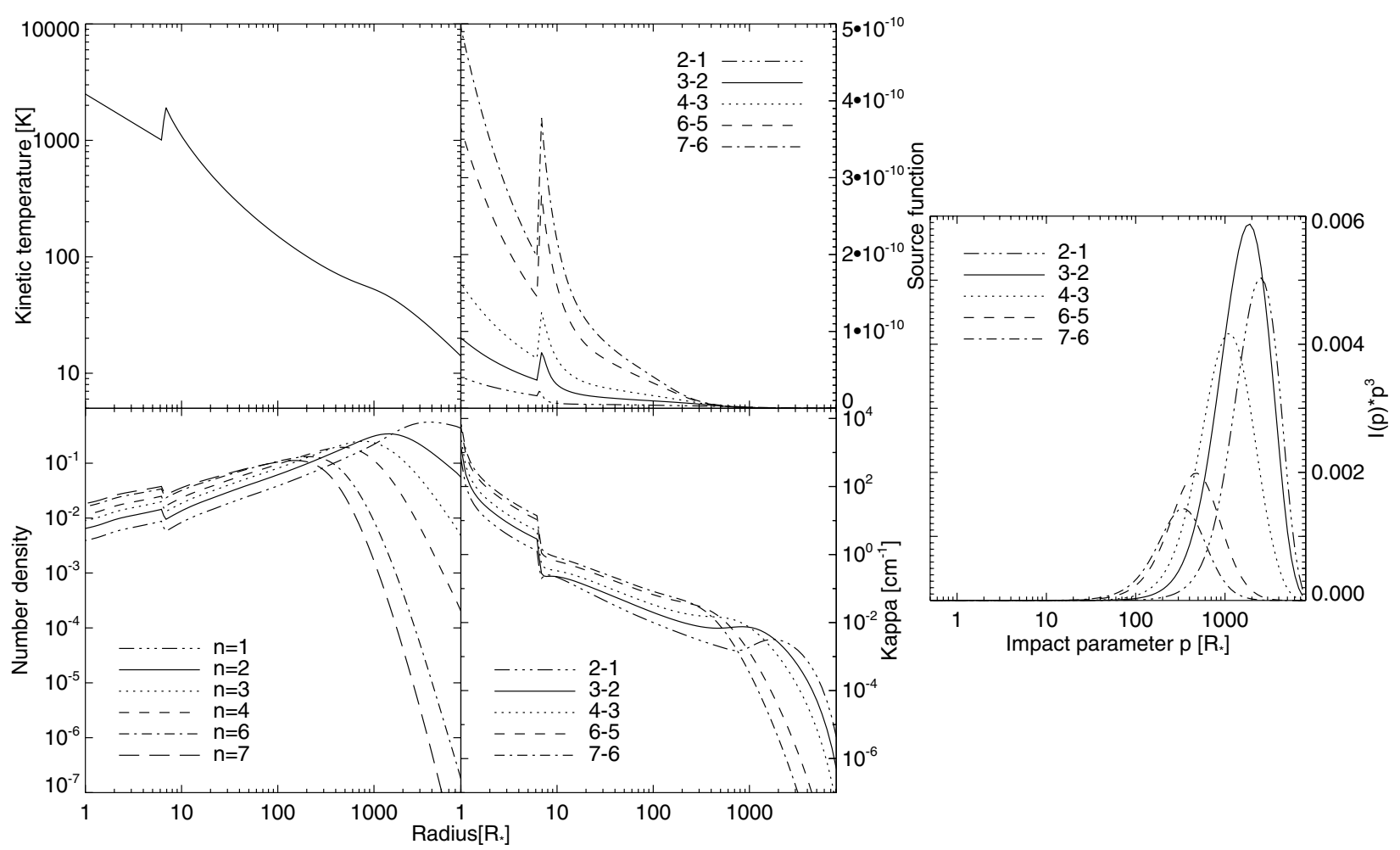

Fig. 5. Detailed look at the wind structure of GX Mon for a constant mass loss of $7.2 \times 10^{-6} M_{\odot} \mathrm{yr}^{-1}$. Temperature [K], normalised number density $\left[\mathrm{cm}^{-3}\right]$, source function [erg s $\left.\mathrm{em}^{-1} \mathrm{~cm}^{-1} \mathrm{ster}^{-1}\right]$, and the absorption coefficient $\kappa_{v}\left[\mathrm{~cm}^{-1}\right]$ as a function of radius; $I(p) * p^{3}$ as function of the impact parameter $p$ - using $T(r)$ and $v(r)$ of Fig. 1.

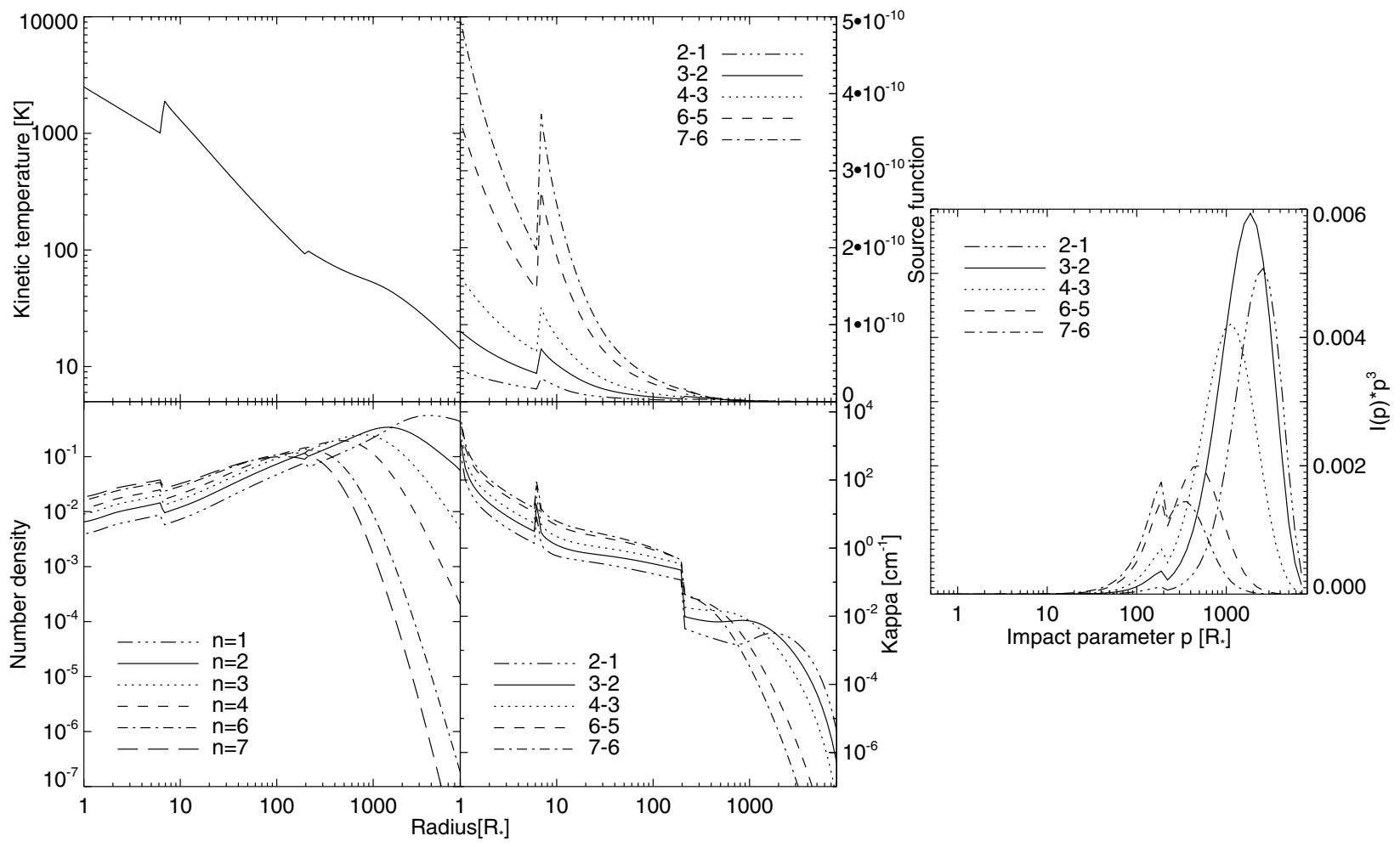

Fig. 6. Detailed look at the wind structure of a superwind phase for GX Mon. The superwind increases the mass loss by a factor 10 from the stellar surface up to $200 R_{\star}$. Temperature $[\mathrm{K}]$, normalised number density $\left[\mathrm{cm}^{-3}\right]$, source function $S_{v}\left[\mathrm{erg} \mathrm{s}^{-1} \mathrm{~cm}^{-2} \mathrm{~Hz}^{-1} \mathrm{ster}^{-1}\right]$, and the absorption coefficient $\kappa_{v}\left[\mathrm{~cm}^{-1}\right]$ as a function of radius; $I(p) * p^{3}$ as function of the impact parameter $p$.

$8.0^{\prime \prime}$ in the $\mathrm{CO}(6-5)$ and $6.0^{\prime \prime}$ in the $\mathrm{CO}(7-6)$ line) the $\mathrm{CO}$ line profiles can not be used to trace small-scale structure, but only to estimate the density, temperature and velocity structure averaged over all directions in the regions where they are formed. 
Table 2. Parameters of VY CMa. We have assumed cosmic carbon and oxygen abundances (Anders \& Grevesse 1989).

\begin{tabular}{lll}
\hline \hline Parameter & This work & Smith et al. (2001) \\
\hline$T_{\star}$ & $2800 \mathrm{~K}$ & $3000 \mathrm{~K}$ \\
$R_{\star}$ & $1.6 \times 10^{14} \mathrm{~cm}$ & $2 \times 10^{13} \mathrm{~cm}$ \\
$L_{\star}$ & $3 \times 10^{5} L_{\odot}$ & $1.6 \times 10^{5} L_{\odot}$ \\
$M_{\star}$ & $15 M_{\odot}$ & $15 M_{\odot}$ \\
$R_{\text {inner }}$ & $10 R_{\star}$ & $7 R_{\star}$ \\
$\dot{M}$ & see Fig. 7 & $10^{-5} \times 10^{-4} M_{\odot} \mathrm{yr}^{-1}$ till $0.5^{\prime \prime}$ \\
$\dot{M}$ & see Fig. 7 & $3 \times 10^{-4} M_{\odot} \mathrm{yr}^{-1}$ from $0.5^{\prime \prime}$ up to $7^{\prime \prime}$ \\
$v_{\text {stoch }}$ & $1 \mathrm{~km} \mathrm{~s}{ }^{-1}$ & $1 \mathrm{~km} \mathrm{~s}^{-1}$ \\
$v_{\infty}$ & $35 \mathrm{~km} \mathrm{~s}^{-1}$ & $35 \mathrm{~km} \mathrm{~s}^{-1}$ \\
distance & $1500 \mathrm{pc}$ & $1500 \mathrm{pc}^{-1}$ \\
$\psi$ & 0.002 & 0.01 \\
\hline
\end{tabular}

Moreover, Smith et al. (2001) demonstrated that the apparent large-scale asymmetry of the nebula in the optical and near-IR images results from a combination of high extinction and backscattering by dust grains. Thermal-infrared images $(5-10 \mu \mathrm{m})$ reveal a more symmetric distribution.

\subsection{Modelling the rotational CO line profiles}

To determine the mass-loss history of VY CMa a first initial grid of $\sim 20000$ models, with stellar parameters as specified in Col. 2 in Table 2, was run to find the global minima in parameter-space using a classical reduced $\chi^{2}$-method. In this, we treat the integrated strength of the line and the line shape as two separate entities to be fitted. This allows to give more weight to the line profile which is more reliable than the absolute flux calibration. The mass-loss parameters of the best models were then further refined.

Parameters characterising the CSE of the best model are given in the second column of Table 2 . Since the calibration uncertainty on the $\mathrm{CO}(2-1)$ and (3-2) line is smaller than for the other profiles, highest weight is given automatically to these lines in the reduced $\chi^{2}$-test. The derived temperature profile, velocity structure and mass-loss history are displayed in Fig. 7; a comparison between observed and theoretical line profiles is shown in Fig. 8.

Before comparing to results of other studies, we first discuss the derived $\dot{M}$-profile and the associated uncertainties. The $\dot{M}$-profile has a value of $8 \times 10^{-5} M_{\odot} \mathrm{yr}^{-1}$ till $80 R_{\star}(\mathrm{de}-$ noted as (A) in Fig. 7). Mass-loss rates in the order of $\sim 1 \times$ $10^{-4} M_{\odot} \mathrm{yr}^{-1}$ in these inner regions still result in acceptable line profiles for the $\mathrm{CO}(6-5)$ and (7-6) line. Between $\sim 80$ and $\sim 600 R_{\star}$, we determine a low mass-loss rate, being of the order of $1-3 \times 10^{-6} M_{\odot} \mathrm{yr}^{-1}$ (denoted as (B) in Fig. 7). This massloss value can not be below $5 \times 10^{-7} M_{\odot} \mathrm{yr}^{-1}$, since the total dust content at the base of the wind would be too low to drive the stellar wind. This period of low mass-loss rate was preceded (in time) by a short period (between 80 and $100 \mathrm{yr}$ at a expansion velocity of $35 \mathrm{~km} \mathrm{~s}^{-1}$ ) of very strong mass loss in the order of 3-3.5 $\times 10^{-4} M_{\odot} \mathrm{yr}^{-1}$ (region (C)). This period is situated around $680 R_{\star}(\sim 1000 \mathrm{yr}$ ago $)$. One can simulate almost analogous line profiles with a somewhat smaller $\dot{M}$ (around $2.5 \times$ $10^{-4} M_{\odot} \mathrm{yr}^{-1}$ ) having lasted somewhat longer ( $\left.150 \mathrm{yr}\right)$. This period of strong mass loss can however not be placed closer to the star, since the predicted $\mathrm{CO}(6-5)$ line becomes far too bright. The estimated mass-loss rate in region (D) is $\sim 1 \times 10^{-6} M_{\odot} \mathrm{yr}^{-1}$.

The estimated kinetic temperature, velocity and mass-loss profile yield the predicted CO line profiles displayed in Fig. 8. The central peak (with a width of $\sim 20 \mathrm{~km} \mathrm{~s}^{-1}$ ) visible in all line profiles originates close to the star $\left(r<15 R_{\star}\right)$, where the dust is accelerated away due to radiation pressure. Region (A) is responsible for the main contribution to the high-excitation $\mathrm{CO}(7-6)$ and (6-5) lines. With a temperature above $\sim 100 \mathrm{~K}$, the density in this region results in an optically-thick, non-resolved Gaussian line profile (see dashed line in Fig. 9). The influence of region (B) is only marginal, and results in a small intensity enhancement in all line profiles (see dashed-dotted line in Fig. 9). The region with a high density enhancement, denoted as region (C), and with an extent from $\sim 600$ to $\sim 700 R_{\star}$, contributes differently to the high-excitation $\mathrm{CO}(7-6)$ and (6-5) lines than to the lower-excitation lines. In the former case, the temperature $(<90 \mathrm{~K})$ in region $(\mathrm{C})$ is too low to significantly populate the higher-excitation energy levels, yielding an optically thin, resolved double-peaked line profile, for the beamwidths of the JCMT. The situation is markedly different for the lowerexcitation lines: (1) the low temperature in this region causes $\sim 5 \%$ of the total $\mathrm{CO}$ number density to be in the ground state, $\sim 13 \%$ to be in the $J=1$ state, $\sim 21 \%$ in the $J=2$ state and $\sim 23 \%$ in the $J=3$ state, yielding optical depths larger than 1 . The shape of the line profiles then follows the shape of the source function $S_{v}$, which form can be directly inferred from the temperature distribution displayed in the upper panel of Fig. 7. The increase and subsequent decrease in $T(r)$ in region $(C)$ results in a double-peaked line profile, with widths in the red and blue peak of some $20 \mathrm{~km} \mathrm{~s}^{-1}$. As an illustration, we have plotted the contribution of region $(\mathrm{A})+(\mathrm{B})+(\mathrm{C})$ to the full line profile in Fig. 9. Note that only the (3-2) line transition is slightly resolved in region $(\mathrm{C})$, while the larger beamwidths for the (2-1) and (1-0) line do not resolve the envelope. It can be seen in Fig. 9 that the intensity in the central peak is under-predicted in the $\mathrm{CO}(2-1)$ and (3-2) line (dotted line in Fig. 9). The low quality of the $\mathrm{CO}(1-0)$ line makes it difficult to substantiate this for this line. The contribution from region (D) fills in this gap, due to the still substantial mass-loss rate of $\dot{M}(r)=1 \times 10^{-6} \dot{M} \mathrm{yr}^{-1}$ in this region and its low temperature.

The small scale ripples visible in the calculated line profiles are not due to numerical noise in the radiative transfer and can not be removed by increasing the number of grid-points (now taken to 150 gridpoints). The cause for the presence of these ripples is the complex temperature structure in region $(\mathrm{C})$ which results from the competing effects of heating and cooling processes. The associated source function is therefore quite complex. In combination with small scale structure in the opacity resulting from the number-density profile this explains these ripples in the theoretical profiles. However, a less abrupt $\dot{M}$-profile would cause these ripples to decrease in amplitude.

The match between observations and theoretical predictions is not perfect. Main reasons for this are the simplifying assumption of a spherical symmetric envelope and a velocity structure being continuously increasing with $r$, as required by the comoving frame formalism. In reality the higher mass-loss rate in region (C) should drive a dusty wind with a different velocity than in the low-mass regions and maybe also with another dust-to-gas ratio.

\subsection{Comparison with other studies}

A direct comparison with other derived mass-loss rates (see Table 3 ) is difficult, since all of the studies mentioned in Table 3 assume a density varying as $r^{-2}$. In case of the analysis of dust spectra, one moreover has to assume a dust-to-gas ratio $\psi$ to estimate the gas mass-loss rate. The main differences with our study are that in our case (1) the mass-loss rate is variable, and hence 
Table 3. Mass-loss rates for VY CMa as found in literature and scaled to a distance of $1500 \mathrm{pc}$.

\begin{tabular}{|c|c|c|c|c|}
\hline & Observ. data & $\dot{M}\left[M_{\odot} \mathrm{yr}^{-1}\right]$ & comments & Reference \\
\hline \multirow{3}{*}{ 总 } & $\mathrm{OH}$ maser emission & $1.2 \times 10^{-4}$ & from $\mathrm{OH}$ shell radius $+n_{\mathrm{H}}=10^{4} \mathrm{~cm}^{-3}$ & Booth et al. (1984) \\
\hline & $\mathrm{CO}(2-1)$ & $1 \times 10^{-4}$ & $\begin{array}{l}\text { scaling based on analysis of } \\
\text { Knapp \& Morris (1985) }\end{array}$ & Zuckerman \& Dyck (1986) \\
\hline & $\mathrm{CO}(1-0)$ & $2.3 \times 10^{-5}$ & from analytical expression & Loup et al. (1993) \\
\hline \multirow{5}{*}{$\stackrel{\overline{\mathscr{D}}}{\vec{\partial}}$} & $\begin{array}{l}\text { spectrosc. data } 2-2.5 \mu \mathrm{m}+ \\
\text { photometr. data till } 20 \mu \mathrm{m}\end{array}$ & $2.3 \times 10^{-4}$ & $\begin{array}{l}\text { data from Hyland et al. (1972); } \\
\psi=0.01\end{array}$ & Bowers et al. (1983) \\
\hline & submm data at $400 \mu \mathrm{m}$ & & $\dot{M}_{\text {dust }}=7.8 \times 10^{-7} M_{\odot} \mathrm{yr}^{-1}$ & Sopka et al. (1989) \\
\hline & ISI $11 \mu \mathrm{m}$ interf. & $3.1 \times 10^{-4}$ & $\psi=0.005$ & Danchi et al. (1994) \\
\hline & spectrosc. data $2.38-200 \mu \mathrm{m}$ & $4 \times 10^{-4}$ & $\psi=0.01$ & Harwit et al. (2001) \\
\hline & surface photometry & $\begin{array}{l}10^{-5}-10^{-4} \\
3 \times 10^{-4}\end{array}$ & $\begin{array}{l}\text { till } 0.5^{\prime \prime} \\
\text { from } 0.5^{\prime \prime} \text { up to } 7^{\prime \prime} \quad \psi=0.01\end{array}$ & Smith et al. (2001) \\
\hline
\end{tabular}

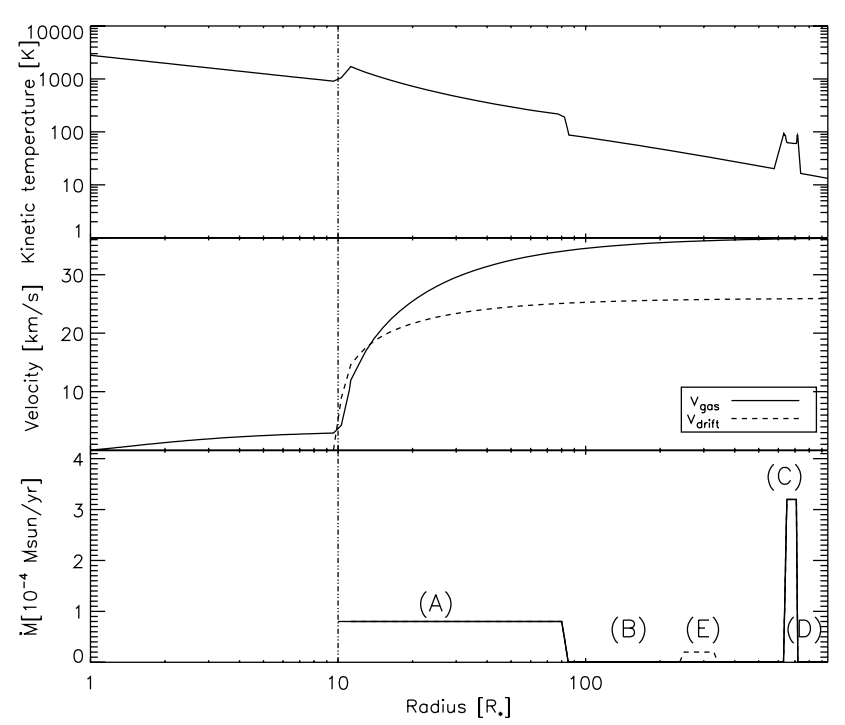

Fig. 7. Upper: estimated temperature profile, middle: estimated velocity structure, and bottom: estimated mass-loss rate $\dot{M}(r)$ for VY CMa as a function of radial distance to the star (black line). The blue/grey line in the panel displaying the mass-loss history represents a model with an extra enhancement in mass loss $\left(\dot{M}(r)=2 \times 10^{-5} M_{\odot} \mathrm{yr}^{-1}\right)$ around $300 R_{\star}$ having the same theoretical line profiles as in Fig. 8 .

the density is not varying as $r^{-2}$; (2) the dust-to-gas ratio is derived self-consistently to obtain the observed expansion velocity; and (3) the IR emission traces warm dust close to the star, while the analysis of different low-excitation rotational CO lines gives us diagnostics on a much broader spatial region.

Assuming a constant mass-loss rate, the best fit model is obtained at $\dot{M}=3.3 \times 10^{-5} M_{\odot} \mathrm{yr}^{-1}$ (see Fig. 10). From the discrepancy in strength of the higher-excitation rotational CO line transitions, it is immediately clear that a model with constant mass loss is unable to explain the observed lines. Moreover, this model fails completely in fitting the detailed line profiles. This is not surprising in view of what is already known about this object (see Sect. 4.3). This value for $\dot{M}$ can be directly compared to the result of Loup et al. (1993), who derived a value of $2.3 \times 10^{-5} M_{\odot} \mathrm{yr}^{-1}$ (scaled to a distance of $1500 \mathrm{pc}$ ) from the CO(1-0) integrated line intensity. Zuckerman \& Dyck (1986) used the $\mathrm{CO}(2-1)$ integrated line intensity as observed with the NRAO $12 \mathrm{~m}$ Kitt Peak to derive a much higher mass-loss rate of $\sim 10^{-4} M_{\odot} \mathrm{yr}^{-1}$. The noise in their observed line profiles was so large that the clear composite nature as seen in Fig. 8 is not traced. As Zuckerman \& Dyck (1986) stated, they scaled the analysis of Knapp \& Morris (1985) for other stars
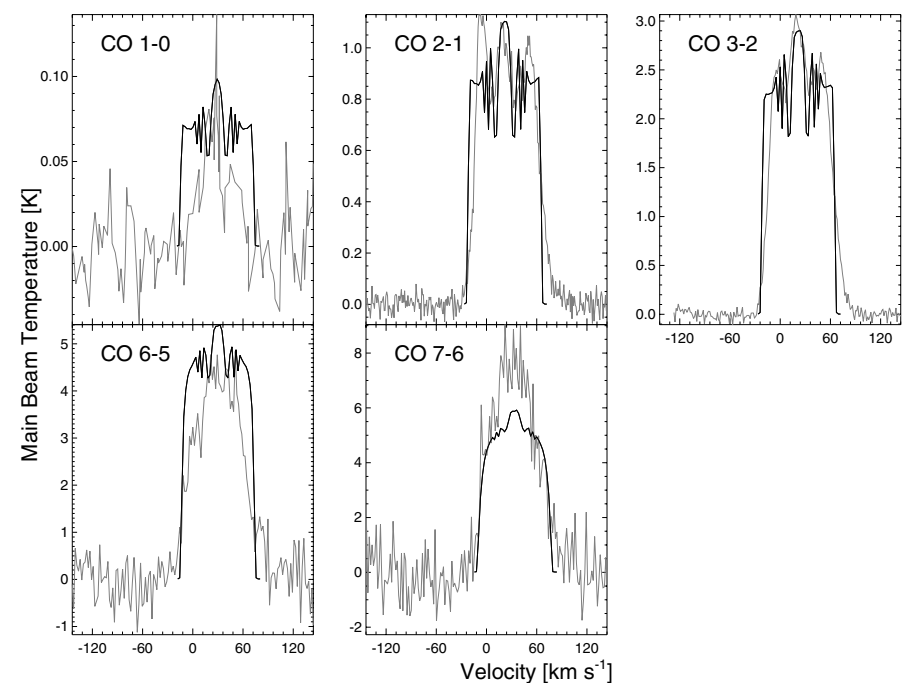

Fig. 8. CO rotational line profiles of VY CMa (Kemper et al. 2003) (grey line) compared with the model predictions (black line) based on the parameters of our best model (see Table 2).

that are located at comparable distances from Earth $(\sim 1500 \mathrm{pc})$. However, Knapp \& Morris (1985) derived mass-loss rates from $\mathrm{CO}(1-0)$ lines of a sample of 50 stars, of which only 2 were supergiants. A key point resulting from our study is that the analysis of full line profiles with different excitation energies provide a much better diagnostics to derive the mass-loss rate than peak intensities, also taking into account that the main calibration uncertainties do not effect the line shape.

Values for the mass-loss rate traced by dust characteristics are generally larger than our mean mass-loss rate of $5.1 \times$ $10^{-5} M_{\odot} \mathrm{yr}^{-1}$, especially keeping in mind that the dust-togas ratio used by other authors (see Table 3 ) is always higher than the value we infer from the CO-lines $(\psi=0.002)$. However, (near)-infrared dust emission traces regions closer to the star than the low-excitation rotational CO lines. The mean mass-loss rate up to $100 R_{\star}$ that we determine is indeed about $0.75( \pm 0.25) \times 10^{-4} M_{\odot} \mathrm{yr}^{-1}$, substantially higher than the mean mass-loss rate averaged over the full envelope of $5.1 \times 10^{-5} M_{\odot} \mathrm{yr}^{-1}$.

\subsection{Discussion: mass-loss variability}

Smith et al. (2001) also studied mass-loss variability. From surface photometry they derived that the star may have experienced enhanced mass loss over the past $1000 \mathrm{yr}\left(\sim 3 \times 10^{-4} M_{\odot} \mathrm{yr}^{-1}\right.$ 

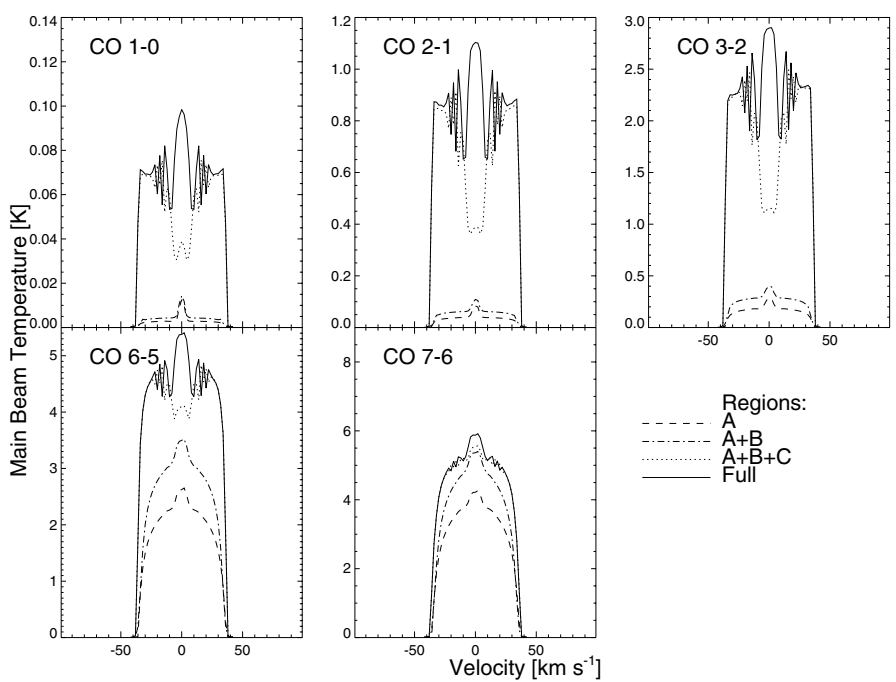

Fig. 9. CO rotational line profiles resulting from the best model (full line) compared to theoretical $\mathrm{CO}$ line profiles taking into account (1) region (A): dashed line, (2) regions $(\mathrm{A})+(\mathrm{B})$ : dashed-dotted line, and (3) regions $(\mathrm{A})+(\mathrm{B})+(\mathrm{C})$ : dotted line.
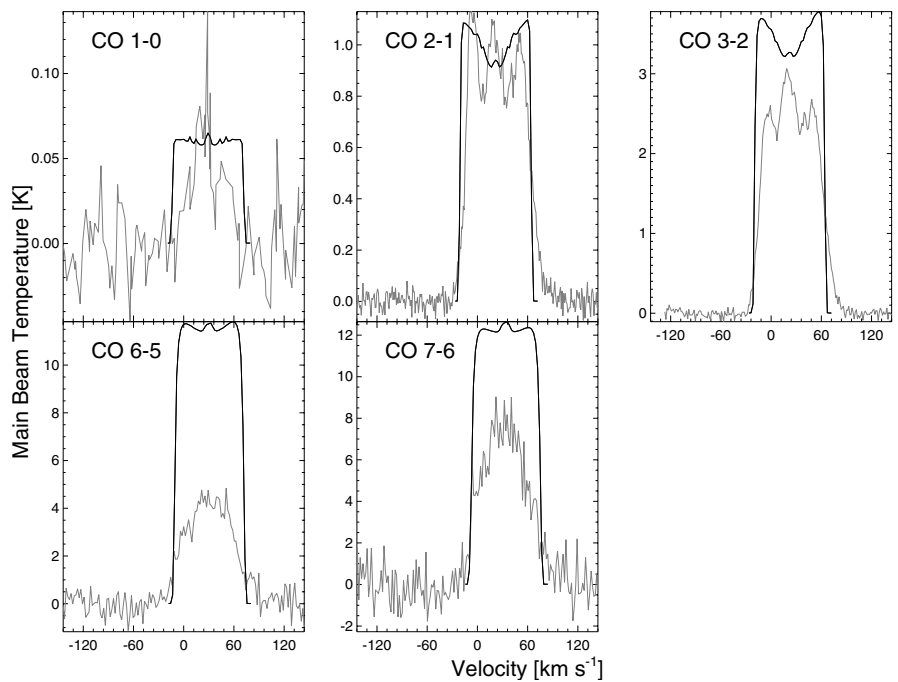

Fig. 10. CO rotational line profiles of VY CMa (Kemper et al. 2003; Nyman et al. 1992) (grey line) compared with the model predictions (black line) based on the stellar parameters of VY CMa as given in the second column of Table 2 , but with a constant $\dot{M}$ of $3.3 \times 10^{-5} M_{\odot} \mathrm{yr}^{-1}$ (black line). The derived dust-to-gas ratio $\psi$ is 0.002 .

between $\sim 0.5^{\prime \prime}$ and $\sim 7^{\prime \prime}$ ), with indications of a lower mass loss (in the order of $10^{-5}$ to $10^{-4} M_{\odot} \mathrm{yr}^{-1}$ till $\sim 0.5^{\prime \prime}$ ) during the past $100 \mathrm{yr}$ (see third column in Table 2). They noted that these estimates can be off by a factor of a few due to uncertainties in the calibration of the mid-IR imaging data, model assumptions and assumed dust properties. Linking our derived mass-loss history with their optical and near infrared images, the enhanced gas density traced in our region (A) coincides with several bright nebular condensations located at $1^{\prime \prime}$ to $2^{\prime \prime}$ south and southwest of the star (indicated by "S" and "SW" in their Fig. 8). The high mass-loss phase indicated by (C) in the bottom panel in Fig. 7 is situated at the same radial distance as their "arc \#1", being from $5^{\prime \prime}$ to 7 " from the star. The two other arcs showing up in their Fig. 8 (named as "arc \#2" and "curved nebulous tail") at $2^{\prime \prime}$ to $4^{\prime \prime}$ from the star, are not directly traced in our mass-loss history. However, test computations show that an extra enhancement of mass loss around $3^{\prime \prime}$ with $\dot{M} \leq 2 \times 10^{-5} M_{\odot} \mathrm{yr}^{-1}$ with an extent of $1^{\prime \prime}$ may be present without seriously altering the calculated line profiles (see dashed line in Fig. 7, indicated by (E)). Hence, we may conclude that our results are consistent with the Hubble and near-IR images presented Smith et al. (2001).

We conclude from our analysis and the study performed by Smith et al. (2001), that VY CMa underwent large changes in mass-loss rate in the order of a factor 100 - averaged over all directions - with a time separation of some $800 \mathrm{yr}$. This timeline is of the same order of magnitude as the separations of 200-800 yr observed by Mauron \& Huggins (1999) observed in the carbonrich AGB-star IRC +10 216. Also Schöier et al. (2005) found that the mass-loss rate producing the faster moving wind in some carbon stars with detached shells should be almost two orders of magnitude higher than the slower AGB wind preceding this violent event. It is important to remark that not all dust-driven winds do display this kind of modulations: a sample of nine carbon-rich AGB-stars with mass-loss larger than $1.5 \times 10^{-5} M_{\odot} \mathrm{yr}^{-1}$ analysed by Schöier et al. (2002) are not likely to have experienced any drastic long-term mass-loss rate modulations over the past thousands of years. As stated already in the introduction, this modulation time scale for AGB stars is too large to be due to stellar pulsations, and too short to be due to nuclear thermal pulses. It is suggested by Simis et al. (2001) that such shells are formed by a hydrodynamical oscillation in the envelope, while the star is on the AGB. The cause of this effect is a subtle mechanism, involving an intricate nonlinear interplay between gas-grain drift, grain nucleation, radiation pressure, and envelope hydrodynamics. Another mechanism proposed by Soker (2000) is a solar-like magnetic activity cycle. The periodic change of direction of the stellar magnetic field enables periodic structure formation, with time intervals between consecutive ejection events in the order of 200-1000 yr. In combination with giant convection cells which may be present in the outer atmospheric layers of red supergiants (Freytag et al. 2002), this may explain the morphology of the arcs (Smith et al. 2001).

\section{Summary}

We have developed a code to derive the gas mass-loss history from a non-LTE analysis of the full line profiles of $\mathrm{CO}$ rotational transitions with different excitation energies, including a selfconsistent computation of the velocity structure and gas kinetic temperature, taking several heating and cooling mechanisms in the CSE into account. The code has been benchmarked to other existing codes, demonstrating good performance.

The code is applied to the analysis of the wind structure of VY CMa. For the first time, we could pinpoint the mass-loss history of this supergiant from the analysis of the $\mathrm{CO}(1-0),(2-1)$, $(3-2),(6-5)$, and (7-6) line profiles and could demonstrate that the mass-loss rate underwent substantial changes during the last $1400 \mathrm{yr}$. We especially note a very strong peak of mass loss of $\sim 3.2 \times 10^{-4} M_{\odot} \mathrm{yr}^{-1}$ some $1000 \mathrm{yr}$ ago having lasted $\sim 100 \mathrm{yr}$. This period of high mass loss was preceded by a long period of low mass loss, in the order of $1 \times 10^{-6} M_{\odot} \mathrm{yr}^{-1}$ taking some $800 \mathrm{yr}$. The current mass loss is estimated to be in the order of $5 \times 10^{-5}$ to $1 \times 10^{-4} M_{\odot} \mathrm{yr}^{-1}$. The mass-loss history that we derive is significantly different from what one would estimate from a constant mass-loss rate model, and therefore paints a much more complete picture on the evolution of the mass loss of AGB and red supergiant stars.

Acknowledgements. L.D. acknowledges financial support from the Fund for Scientific Research - Flanders (Belgium), SH acknowledges financial 
support from the Interuniversity Attraction Pole of the Belgian Federal Science Policy P5/36. We would like to thank our colleagues Prof. P. Dierckx and Prof. R. Piessens from the Department of Computer Science (KULeuven) for their useful advice.

\section{References}

Anders, E., \& Grevesse, N. 1989, Geochim. Cosmochim. Acta, 53, 197 Bakes, E. L. O., \& Tielens, A. G. G. M. 1994, ApJ, 427, 822

Biermann, P., \& Harwit, M. 1980, ApJ, 241, L105

Booth, R. S., Diamond, P. J., \& Norris, R. P. 1984, in VLBI and Compact Radio Sources, IAU Symp., 110, 313

Bowers, P. F., Johnston, K. J., \& Spencer, J. H. 1983, ApJ, 274, 733

Burke, J. R., \& Hollenbach, D. J. 1983, ApJ, 265, 223

Castor, J. I. 1970, MNRAS, 149, 111

Che, A., Hempe, K., \& Reimers, D. 1983, A\&A, 126, 225

Danchi, W. C., Bester, M., Degiacomi, C. G., Greenhill, L. J., \& Townes, C. H. 1994, AJ, 107, 1469

de Jong, T. 1977, A\&A, 55, 137

Dominik, C., Gail, H.-P., \& Sedlmayr, E. 1989, A\&A, 223, 227

Draine, B. T., \& Lee, H. M. 1984, ApJ, 285, 89

Efstathiou, A., \& Rowan-Robinson, M. 1990, MNRAS, 245, 275

Fong, D., Meixner, M., \& Shah, R. Y. 2003, ApJ, 582, L39

Freytag, B., Steffen, M., \& Dorch, B. 2002, Astron. Nachr., 323, 213

Goldreich, P., \& Scoville, N. 1976, ApJ, 205, 144

Goldsmith, P. F., \& Langer, W. D. 1978, ApJ, 222, 881

Goorvitch, D., \& Chackerian, C. 1994, ApJS, 91, 483

Green, S., Maluendes, S., \& McLean, A. D. 1993, ApJS, 85, 181

Groenewegen, M. A. T. 1994, A\&A, 290, 531

Habing, H. J., \& Olofsson, H. (eds.) 2003, Asymptotic giant branch stars (New York, Berlin: Springer)

Harrington, J. P., \& Borkowski, K. J. 1994, Bull. Am. Astron. Soc., 26, 1469

Harwit, M., Malfait, K., Decin, L., et al. 2001, ApJ, 557, 844

Herbig, G. H. 1972, ApJ, 172, 375

Hillier, D. J. 1990, A\&A, 231, 116

Hollenbach, D., \& McKee, C. F. 1979, ApJS, 41, 555

Hollenbach, D., \& McKee, C. F. 1989, ApJ, 342, 306

Hollenbach, D. J., \& Tielens, A. G. G. M. 1999, Rev. Mod. Phys., 71, 173

Huggins, P. J., \& Glassgold, A. E. 1982, AJ, 87, 1828

Hyland, A. R., Becklin, E. E., Frogel, J. A., \& Neugebauer, G. 1972, A\&A, 16, 204

Iben, I., \& Renzini, A. 1983, ARA\&A, 21, 271

Justtanont, K., Skinner, C. J., \& Tielens, A. G. G. M. 1994, ApJ, 435, 852

Justtanont, K., Skinner, C. J., Tielens, A. G. G. M., Meixner, M., \& Baas, F. 1996, ApJ, 456, 337

Justtanont, K., \& Tielens, A. G. G. M. 1992, ApJ, 389, 400

Kemper, F., Stark, R., Justtanont, K., et al. 2003, A\&A, 407, 609

Knapp, G. R., \& Morris, M. 1985, ApJ, 292, 640

Knapp, G. R., Phillips, T. G., \& Huggins, P. J. 1980, ApJ, 242, L25

Knapp, G. R., Phillips, T. G., Leighton, R. B., et al. 1982, ApJ, 252, 616

Kwok, S. 1975, ApJ, 198, 583
Larsson, B., Liseau, R., \& Men'shchikov, A. B. 2002, A\&A, 386, 1055

Le Sidaner, P., \& Le Bertre, T. 1996, A\&A, 314, 896

Loup, C., Forveille, T., Omont, A., \& Paul, J. F. 1993, A\&AS, 99, 291

Mamon, G. A., Glassgold, A. E., \& Huggins, P. J. 1988, ApJ, 328, 797

Marshall, J. R., van Loon, J. T., Matsuura, M., et al. 2004, MNRAS, 355, 1348

Mathis, J. S., Rumpl, W., \& Nordsieck, K. H. 1977, ApJ, 217, 425

Mauron, N., \& Huggins, P. J. 1999, A\&A, 349, 203

Mauron, N., \& Huggins, P. J. 2000, A\&A, 359, 707

McKee, C. F., Storey, J. W. V., Watson, D. M., \& Green, S. 1982, ApJ, 259, 647

Mihalas, D., Kunasz, P. B., \& Hummer, D. G. 1975, ApJ, 202, 465

Monnier, J. D., Tuthill, P. G., Lopez, B., et al. 1999, ApJ, 512, 351

Morris, M. 1980, ApJ, 236, 823

Nejad, L. A. M., \& Millar, T. J. 1988, MNRAS, 230, 79

Netzer, N., \& Knapp, G. R. 1987, ApJ, 323, 734

Ney, E. P., Merrill, K. M., Becklin, E. E., Neugebauer, G., \& Wynn-Williams, C. G. 1975, ApJ, 198, L129

Nyman, L.-A., Booth, R. S., Carlstrom, U., et al. 1992, A\&AS, 93, 121

Phillips, T. R., Maluendes, S., \& Green, S. 1996, ApJS, 107, 467

Reid, M. J., \& Dickinson, D. F. 1976, ApJ, 209, 505

Reid, M. J., \& Muhleman, D. O. 1978, ApJ, 220, 229

Rodgers, B., \& Glassgold, A. E. 1991, ApJ, 382, 606

Rothman, L. S., Gamache, R. R., Goldman, A., et al. 1987, Appl. Opt., 26, 4058

Sahai, R., Trauger, J. T., Watson, A. M., et al. 1998, ApJ, 493, 301

Schinke, R., Engel, V., Buck, U., Meyer, H., \& Diercksen, G. H. F. 1985, ApJ, 299, 939

Schöier, F. L., Lindqvist, M., \& Olofsson, H. 2005, A\&A, 436, 633

Schöier, F. L., Ryde, N., \& Olofsson, H. 2002, A\&A, 391, 577

Schönberg, K. 1985, A\&A, 148, 405

Schönberg, K. 1988, A\&A, 195, 198

Schönberg, K., \& Hempe, K. 1986, A\&A, 163, 151

Schutte, W. A., \& Tielens, A. G. G. M. 1989, ApJ, 343, 369

Simis, Y. J. W., Icke, V., \& Dominik, C. 2001, A\&A, 371, 205

Smith, N., Humphreys, R. M., Davidson, K., et al. 2001, AJ, 121, 111

Snyder, L. E., \& Buhl, D. 1975, ApJ, 197, 329

Sobolev, V. 1947, Moving Envelopes of Stars (Cambridge Harvard University Press)

Soker, N. 2000, ApJ, 540, 436

Sopka, R. J., Olofsson, H., Johansson, L. E. B., Nguyen, Q.-R., \& Zuckerman, B. 1989, A\&A, 210, 78

Tielens, A. G. G. M. 1983, ApJ, 271, 702

Tielens, A. G. G. M., \& Hollenbach, D. 1985, ApJ, 291, 722

Truong-Bach, Morris, D., \& Nguyen-Q-Rieu. 1991, A\&A, 249, 435

van Loon, J. T., Marshall, J. R., Matsuura, M., \& Zijlstra, A. A. 2003, MNRAS, 341,1205

van Zadelhoff, G.-J., Dullemond, C. P., van der Tak, F. F. S., et al. 2002, A\&A, 395,373

Winters, J. M., Le Bertre, T., Jeong, K. S., Nyman, L.-Å., \& Epchtein, N. 2003, A\&A, 409, 715

Wittkowski, M., Langer, N., \& Weigelt, G. 1998, A\&A, 340, L39

Zuckerman, B., \& Dyck, H. M. 1986, ApJ, 304, 394

Zuckermann, B., Palmer, P., Morris, M., et al. 1977, ApJ, 211, L97 